\title{
Link Reliability Based Greedy Perimeter Stateless Routing for Vehicular Ad Hoc Networks
}

\author{
Siddharth Shelly and A. V. Babu \\ Department of Electronics and Communication Engineering, National Institute of Technology Calicut, Calicut 673 601, India
}

Correspondence should be addressed to Siddharth Shelly; sidharthshelly_pec10@nitc.ac.in

Received 16 October 2014; Revised 4 March 2015; Accepted 4 March 2015

Academic Editor: Abdelaziz Bensrhair

Copyright (C) 2015 S. Shelly and A. V. Babu. This is an open access article distributed under the Creative Commons Attribution License, which permits unrestricted use, distribution, and reproduction in any medium, provided the original work is properly cited.

\begin{abstract}
We propose an enhancement for the well-known greedy perimeter stateless routing (GPSR) protocol for vehicular ad hoc networks (VANETs), which exploits information about link reliability when one-hop vehicles are chosen for forwarding a data packet. In the proposed modified routing scheme, a tagged vehicle will select its one-hop forwarding vehicle based on reliability of the corresponding communication link. We define link reliability as the probability that a direct link among a pair of neighbour vehicles will remain alive for a finite time interval. We present a model for computing link reliability and use this model for the design of reliability based GPSR. The proposed protocol ensures that links with reliability factor greater than a given threshold alone are selected, when constructing a route from source to destination. The modified routing scheme shows significant improvement over the conventional GPSR protocol in terms of packet delivery ratio and throughput. We provide simulation results to justify the claim.
\end{abstract}

\section{Introduction}

Vehicular ad hoc networks (VANETs) are poised to be an integral part of intelligent transportation system (ITS) initiatives all over the world. Such intervehicle communication networks support two distinct communication scenarios: vehicle-to-vehicle (V2V) and vehicle-to-infrastructure (V2I) communications [1-4]. The IEEE 802.11p is an approved amendment to the IEEE 802.11 standard for enabling vehicular communications [5]. It specifies the PHY and MAC protocols for wireless access in vehicular environment (WAVE), while higher layer protocols are based on IEEE 1609 standards $[3,5]$.

Ensuring reliable routing is a challenging task in VANETs since vehicles move with very high velocities that result in dynamic network topology. The routes that are established between a source-destination pair may cease to be invalid when at least one communication link along the route fails. The link lifetime is the time duration for which two vehicles are within the communication range of each other. In other words, it is the time period that starts when two vehicles move to the communication range of each other and that ends when they move out of their range (i.e., signal-tonoise ratio perceived by the receiver vehicle becomes less than the minimum required). When a link on a routing path fails, network connectivity properties change rapidly. This results in temporary disruption of information flow and leads to initiation of yet another route discovery process. Route rediscovery is expensive in terms of required signaling and computation overheads. Hence, during route discovery phase, it is very important and desirable for the routing algorithm to choose optimal route connecting source and destination, consisting of the most reliable links in the network [6].

Greedy perimeter stateless routing (GPSR) [7] is a geographic routing protocol that relies on positions (coordinates) of the nodes and destination address of the packet to make forwarding decisions in multihop wireless networks. In GPSR protocol, greedy forwarding is employed to forward packets. Always, a node that is closer to destination is selected as the forwarding node. When greedy forwarding fails, GPSR algorithm will employ perimeter forwarding. Recently many variations of the conventional GPSR protocol have been proposed for VANETs [8-10]. Studies of GPSR conducted in 
[11] suggest that it suffers from many disadvantages especially in VANETs. Due to rapidly changing network topology, a source vehicle may not receive updated position information from its neighbours periodically. Hence, it may make wrong forwarding decisions resulting in failure of greedy forwarding. Perimeter mode forwarding can be used when greedy forwarding fails; however, it leads to sharp increase in delay owing to the higher number of hops required to reach destination.

In this paper, we propose a reliability based GPSR protocol (GPSR-R) for VANETs on highways. In the proposed routing protocol, a tagged vehicle will select its one-hop forwarding vehicle based on reliability of the corresponding communication link. To facilitate this, we use a metric known as link reliability which is defined as the probability that a link will be alive for a finite time duration. The selection of forwarding nodes is executed based on this metric. Thus the proposed protocol ensures that links with reliability factor greater than a given threshold alone are selected when constructing a route from source to destination. Simulation results show that the modified scheme shows improvement over the conventional GPSR protocol. The major contributions of this paper are as follows.

(i) We propose a new analytical model for describing link reliability and derive an analytical expression for computing link reliability. The analysis takes into account free flow uncongested traffic scenario and assumes the vehicle speed to have uniform probability density function.

(ii) We modify the conventional GPSR protocol and design a reliability based GPSR algorithm. We perform detailed evaluation of the modified routing algorithm. Further we compare the performance of GPSR$\mathrm{R}$ against conventional GPSR protocol and three representative reliable VANET routing protocols that exist in the literature and establish that GPSR-R provides more improvement in packet delivery ratio and network throughput.

The rest of this paper is organized as follows. Section 2 describes the related work. Section 3 describes the mathematical model for link reliability. Section 4 describes the reliability based GPSR protocol. The evaluation of the modified routing algorithm is presented in Section 5. The paper is concluded in Section 6.

\section{Related Work}

Several papers have addressed the design of reliable routing algorithm for mobile ad hoc networks (MANETs) [12-15]. Such designs are not applicable to VANETs because of distinct mobility and topology characteristics of these networks. Recently, several papers have appeared that deal with reliable routing in VANETs [16-27]. In [16], Taleb et al. describe a reliable routing protocol in which vehicles are grouped according to their velocity vectors and the routing algorithm dynamically searches for the most stable route that includes only hops from the same group. The performance of the algorithm depends on prediction of link failures prior to their occurrence. Wan et al. [17] propose a reliable routing protocol for V2I networks on rural highways based on prediction of link lifetime. Namboodiri and Gao [18] describe a routing algorithm that predicts how long a route will last and creates new route before the failure of the existing route. In [19], Menouar et al. describe a routing algorithm that can predict the future coordinates of a vehicle and build new stable routes. In [20], the same authors propose a movement prediction based routing (MOPR) in which each vehicle estimates the link stability, a measure of link lifetime, for each neighbouring vehicle before selecting the next hop for data forwarding. Authors of abovementioned papers compute link lifetime by assuming both the intervehicle distance and the velocity to be deterministic quantities. However, as is widely known, both of these quantities are random variables. Sofra and Leung [21] propose an estimation method for link quality in terms of link residual lifetime. The same authors in [22] demonstrate that the estimation method proposed in [21] is capable of finding reliable routes in VANETs. However, calculation of residual lifetime requires removal of noise from the data and estimation of various parameters related to the model. In [23], authors present a protocol called GPSR-L, an improved version of GPSR protocol that takes into account the link lifetime for the selection of next hop forwarding node. However, authors present an oversimplified model for finding the link lifetime by assuming vehicle velocity to be a constant. In [24], Eiza et al. propose a reliable routing protocol known as AODV-R by incorporating link reliability metric in the original AODV routing protocol. In [25], Niu et al. describe a QoS routing algorithm based on AODV protocol and a criterion for link reliability. In [26], Yu et al. present a routing procedure, AODV-VANET, that uses vehicles' movement information in the route discovery process. Notice that the link reliability model employed in $[24,25]$ does not consider the stochastic nature of the intervehicle distance. Further, several studies have reported that topology based routing schemes such as AODV perform badly in VANETs, as compared to geographic routing protocols [6]. In [27], Eiza and Ni propose a routing algorithm that exploits the evolving characteristics of VANETs on highway. Naumov and Gross in [28] propose connectivity aware routing (CAR) in VANETs, which adapts to current network conditions to find a route with sufficient connectivity, so as to maximize the chance of successful packet delivery. In [29], GalavizMosqueda et al. propose a free standing position based routing (FPBR) algorithm that takes into account realistic channel propagation model in its design. In [30], Boukerche et al. describe a routing approach for providing QoS in VANETs in which the link reliability is estimated based on exchange of beacons among vehicles. In [31], Yu et al. propose a routing protocol for VANETs based on vehicle density so as to provide fast and reliable message delivery. In [32], Cai et al. propose a link state aware geographic opportunistic routing protocol (LSGO), in which the forwarding nodes are selected based on their geographic location and the link quality expressed in terms of a metric known as expected transmission count. 
Different from aforementioned category of link stabilitybased routing protocols where the principal objective is to find a reliable packet delivery route between the source and the destination nodes for improving the packet delivery ratio, trajectory-based routing [33-36] relies on the construction of a predefined trajectory between the source and the destination nodes based on the knowledge of the network topology. The source nodes are required to encode a geographical trajectory into the packet header and each intermediate node uses geographical greedy forwarding strategy along the trajectory. However, encoding and storing of trajectory information can limit the protocol scalability, because, for a longer path, the required header size would be very large.

Knowledge of link lifetime and reliability is essential for the design of link reliability based routing protocols. Recently, there have been certain attempts to analyse the link duration and link reliability in VANETs [37-42]. In [37], Sun et al. propose an analytical model for the PDF of link lifetime by assuming equidistant nodes and vehicle speed as Gaussian. However, it may be noted that the intervehicle distance is, in general, a random variable. Yan and Olariu [38] investigate the PDF of the link lifetime in a VANET assuming (i) the PDF of intervehicle headway distance to be log-normal and (ii) the vehicle speed to be deterministic. Rudack et al. [39] present an analytical framework for single-hop link duration in VANETs. Wang [40] presents a simulation study of link duration, route lifetime, and route repair frequency in VANETs. Abboud and Zhuang [41] present a probabilistic analysis of communication link in VANETs for three distinct ranges of vehicle density. In [42], Shelly and Babu present an analysis of link duration in VANETs for the free flow traffic state.

One of the major disadvantages of the GPSR protocol is that while the sender routes the packet to the node closest to the destination node the selected forwarding node can be at the edge of the sender's communication range which can lead to packet loss [43]. In VANETs, the abovementioned problem can be quite severe due to the dynamic characteristics of the network topology. Hence, for a VANET scenario, the conventional GPSR protocol should be modified to ensure that link reliability is also considered when the next hop forwarding vehicle is chosen. In this paper, we, first of all, present an accurate model for link duration and link reliability in VANETs by considering stochastic characteristics of the intervehicle distance and the vehicle speed. Contrary to the link stability model used in $[20,23,32]$ for the selection of one-hop neighbour, the basic approach followed in our paper is that a vehicle initially finds a continuous time period $\left(T_{p}\right)$ in which a currently available link to one of its neighbours will be available from a time $t$. The vehicle then finds the probability that the link would be actually available for the duration $\left(t, t+T_{p}\right)$. In our proposed GPSR enhancement protocol, the neighbor vehicle that satisfies the link reliability criterion alone would be eligible for selection as a forwarding node. Accordingly, the proposed reliability based GPSR protocol ensures that most reliable nodes are chosen for forwarding and for building a route from source to destination. We implement the protocol using NS2 and our extensive simulation results show that the proposed protocol outperforms the conventional GPSR protocol.

\section{Analytical Model for Link Reliability in VANETs}

We now describe a model for link reliability in VANETs.

3.1. System Model. For the analysis of link reliability, we consider the free flow traffic state and assume vehicle arrival process to be Poisson [44-46]. Accordingly, the intervehicle distances are i.i.d exponential with parameter $\rho[44,45]$. In the uncongested free flow traffic state, vehicles move independently of each other in the network. Further, the probability distribution of vehicle speed can be approximated to be uniform [46-48]. Let $V$ be the random variable representing the vehicle speed. Assume $V$ to be uniform in the interval $\left(v_{\min }, v_{\max }\right)$. The PDF of $V$ is then given by

$$
f_{V}(v)=\frac{1}{v_{\max }-v_{\min }} ; \quad v_{\min } \leq v \leq v_{\max } .
$$

Now the cumulative distribution function (CDF) of intervehicle distance $X_{n}$ is given by $F_{X_{n}}(x)=1-e^{-\rho x}, x \geq 0$, where $\rho=\lambda \sum_{i=1}^{M}\left(p_{i} / v_{i}\right)=\lambda E[1 / V][44,45]$. Here $E[\cdot]$ is the expectation operator and $\lambda$ represents the arrival rate. When the vehicle speed follows uniform PDF, the average vehicle density $\rho$ is computed as follows:

$$
\begin{aligned}
\rho & =\lambda \int_{v_{\min }}^{v_{\max }} \frac{1}{v} f_{V}(v) d v \\
& =\frac{\lambda}{v_{\max }-v_{\min }}\left[\log v_{\max }-\log v_{\min }\right] .
\end{aligned}
$$

3.2. Probability Distribution of Link Duration. Here, we determine the probability distribution of link duration in VANETs. Consider the one-dimensional VANET forming a single-lane highway shown in Figure 1, where all the vehicles move in the same direction. All the vehicles on the highway have the same mean velocities, but they are permitted to move with variable instantaneous velocities. We assume a fixed transmission range ( $R$ meters) and a fixed transmission power for all the vehicles. Consider two vehicles $C_{A}$ and $C_{B}$ moving in the network as shown in Figure 1. Even though they have the same speed statistics, their instantaneous velocities are different. Let $V_{A}, V_{B}$, and $V_{r}$, respectively, be the random variables that represent the velocities of vehicle $C_{A}$, vehicle $C_{B}$ and the relative velocity between the given pair of vehicles in the network. Since $V_{r}=V_{A}-V_{B}$, the dynamic range of $V_{r}$ is limited to $\left(-v_{m},+v_{m}\right)$ where $v_{m}=v_{\max }-v_{\min }$. Further, the PDF of $V_{r}, f_{V_{r}}\left(v_{r}\right)$, can be determined by using the principle of random variable transformation and is given by the proof given in Appendix A:

$$
f_{V_{r}}\left(v_{r}\right)= \begin{cases}\frac{v_{r}+v_{\max }-v_{\min }}{\left(v_{\max }-v_{\min }\right)^{2}} ; & -v_{m} \leq v_{r} \leq 0 \\ \frac{v_{\max }-v_{\min }-v_{r}}{\left(v_{\max }-v_{\min }\right)^{2}} ; & 0 \leq v_{r} \leq v_{m} .\end{cases}
$$




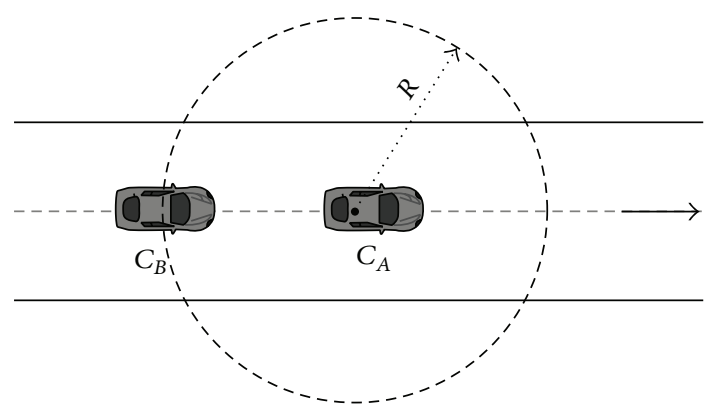

FIGURE 1: Single-lane highway model.

Let $T$ be the link duration, that is, the time duration for which communication link between vehicles $A$ and $B$ is active. Now $T$ is computed as follows:

$$
T=\frac{X}{V_{r}} .
$$

Here $X$ is a random variable that represents the active distance over which vehicles $A$ and $B$ communicate. As described in Section 3.1, since the intervehicle distances are i.i.d and exponential with parameter $\rho=\lambda E[1 / V]$, the PDF of $X$ is given by

$$
f_{X}(x)=\rho e^{-\rho|x|} ; \quad-R \leq x \leq R
$$

Assuming that $X$ and $V_{r}$ are independent, the $\operatorname{CDF}$ of $T, F_{T}(t)$, can be written as follows:

$$
F_{T}(t)=P(T \leq t)=P\left(X \leq V_{r} t\right) .
$$

Using the principle of random variable transformation, $F_{T}(t)$ can be determined as follows (proof given in Appendix B):

$$
F_{T}(t)= \begin{cases}F_{T_{1}}(t) ; & t \leq \frac{R}{v_{m}} \\ F_{T_{1}}(t)-F_{T_{2}}(t) ; & t>\frac{R}{v_{m}},\end{cases}
$$

where the terms $F_{T_{1}}(t)$ and $F_{T_{2}}(t)$ are given as follows:

$$
\begin{array}{r}
F_{T_{1}}(t)=2 \int_{v_{r}=0}^{v_{m}} \int_{x=-R}^{v_{r} t} f_{X, V_{r}}\left(x, v_{r}\right) \mathrm{d} x \mathrm{~d} v_{r}^{(0)} ; \\
t \leq \frac{R}{v_{m}}, \\
F_{T_{2}}(t)=2 \int_{v_{r}=R / t}^{v_{m}} \int_{x=R}^{v_{r} t} f_{X, V_{r}}\left(x, v_{r}\right) \mathrm{d} x \mathrm{~d} v_{r} ; \\
t>\frac{R}{v_{m}} .
\end{array}
$$

Here $f_{X, V_{r}}\left(x, v_{r}\right)$ is the joint PDF of $X$ and $V_{r}$. The PDF of $T$, $f_{T}(t)$, is obtained by differentiating (7a) with respect to $t$ and is given by

$$
f_{T}(t)= \begin{cases}f_{T_{1}}(t) ; & t \leq \frac{R}{v_{m}} \\ f_{T_{1}}(t)-f_{T_{2}}(t) ; & t>\frac{R}{v_{m}} .\end{cases}
$$

Here the two terms $f_{T_{1}}(t)$ and $f_{T_{2}}(t)$ that define $f_{T}(t)$ are computed as follows (details given in Appendix B):

$$
\begin{gathered}
f_{T_{1}}(t)=\rho \frac{\rho t v_{m}\left(e^{-\rho t v_{m}}+1\right)+2\left(e^{-\rho t v_{m}}-1\right)}{v_{m}^{2} \rho^{3} t^{3}} \\
f_{T_{2}}(t)=\frac{1}{v_{m}^{2}}\left(\left(\frac{e^{-\rho t v_{m}}\left(\rho v_{m} t+2\right)}{\rho^{2} t^{3}}\right)-e^{-\rho R} R\left(\frac{v_{m}}{t^{2}}-\frac{R}{t^{3}}\right)\right. \\
\left.+e^{-\rho R}\left(\frac{v_{m}}{\rho t^{2}}-\frac{2 R}{\rho t^{3}}-\frac{2}{\rho^{2} t^{3}}\right)\right) .
\end{gathered}
$$

The average link lifetime is then computed as follows:

$$
E[T]=\int_{t} t f_{T}(t) \mathrm{d} t
$$

Notice that $E[T]$ should be determined by numerical integration procedure.

3.3. An Analytical Model for Link Reliability. In this section, we use the expression for link duration PDF obtained in the previous section to determine the link reliability. We follow the probabilistic link reliability model of [27] in which the link reliability for a link at time $t$ is defined as follows:

$$
\begin{aligned}
& r_{\text {link }}(t) \\
& =\operatorname{Prob}\left(\text { link } i \text { continues to be available until } t+T_{P}\right. \\
& \mid \text { link } i \text { is available at } t),
\end{aligned}
$$

where $T_{P}$ is the duration for which the given link should be available for communication. Given the link duration PDF $f_{T}(t)$, the link reliability is determined as follows (for detailed analytical expressions, refer to Appendix C):

$$
r_{\text {link }}(t)= \begin{cases}\int_{t}^{t+T_{p}} f_{T}(t) \mathrm{d} t ; & T_{p}>0 \\ 0 ; & \text { otherwise. }\end{cases}
$$

The link reliability defined above is a measure of stability of the link and hence a vehicle can use this as a metric for choosing its forwarding node. The most reliable forwarding node, which satisfies the reliability requirements, should be selected by the source node. In the next section we discuss the design of a reliable routing protocol based on this criterion.

Notice that computation of link reliability probability according to (11) involves a vehicle to find a continuous 
time interval $\left(T_{p}\right.$ - the duration vehicle will be connected to its neighbor from a reference time) by assuming that both vehicles associated with the link maintain their current velocity unchanged during $T_{p}$. The vehicle then finds the probability that the link will really last till $t+T_{p}$. It may be noted that the quantity $T_{p}$ in (10) can be defined as the duration for which the communication link between a given pair of vehicles $C_{A}$ and $C_{B}$ is continuously available. To find $T_{p}$, we make the following assumptions. (i) During $T_{p}$, the vehicles associated with the link do not change their velocities and (ii) the highway width is negligible compared to vehicle's communication range. Now $T_{P}$ is computed as follows. If $V_{B} \geq V_{A}$, that is, when vehicle $C_{B}$ approaches vehicle $C_{A}$ from behind, the $T_{p}$ is calculated as $T_{p}=\left(R+L_{A B}\right) / V_{r}$ and if $V_{A} \geq$ $V_{B}$, that is, when vehicle $C_{A}$ moves forward in front of vehicle $C_{B}$, the $T_{p}$ is calculated as $T_{p}=\left(R-L_{A B}\right) / V_{r}$ as shown in Figure 1. Here $L_{A B}$ is the Euclidean distance between the two nodes and is computed as $L_{A B}=\sqrt{\left(y_{A}-y_{B}\right)^{2}+\left(x_{A}-x_{B}\right)^{2}}$. Further we assume that all the vehicles possess the GPS facility to identify their location and velocity. Each node will receive the velocity and position information of its neighbour nodes from the modified beacon structure, which will be explained in Section 4.2. Once these values are obtained, the value of $T_{p}$ can be computed, and the link reliability can be computed by (11).

\section{Reliability Based GPSR Protocol: GPSR-R}

In this section, first of all, we provide a brief overview of the conventional GPSR protocol and then describe the proposed reliability based GPSR protocol (GPSR-R).

4.1. GPSR: An Overview. Geographic based greedy forwarding is one of the most promising routing approaches for VANETs [6]. GPSR [7] is a geographic routing protocol that relies on the location coordinates of the nodes and the destination address of the packet to find the next hop forwarding node. In GPSR, a packet is marked by its originator with the corresponding destination address. Assuming that location coordinates are known, the nodes choose to forward the packet to the one-hop neighbour located closer to the destination. This is continued until the destination is reached. If such greedy forwarding is not possible, GPSR employs perimeter forwarding. The protocol assumes that all the nodes that participate in the data transfer process possess the GPS facility to identify their location coordinate. Nodes periodically exchange beacon messages among themselves that contain their ID (address) and the location coordinates. In the context of one-dimensional VANETs, Figure 2 shows the greedy forwarding method while the perimeter forwarding strategy is shown in Figure 3.

However, in VANETs, GPSR suffers from neighbour wireless link break problem [43]. Because of dynamic network topology, a source vehicle may fail to receive updated position information from its neighbours which are located at the edge of its communication range. Consequently, when the source vehicle uses greedy forwarding, there is a high probability that the selected one-hop forwarding vehicle may have gone out of

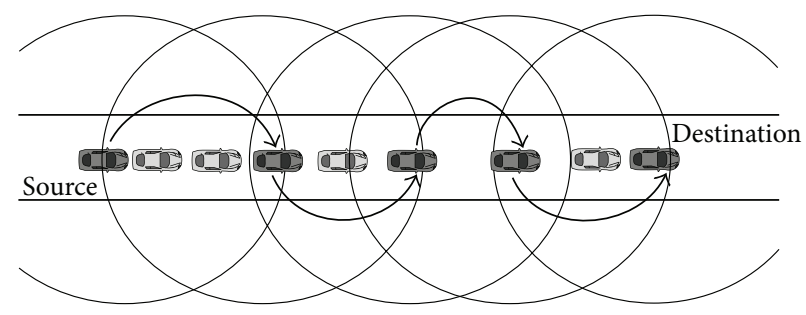

FIGURE 2: Greedy forwarding in GPSR when employed in vehicular networks.

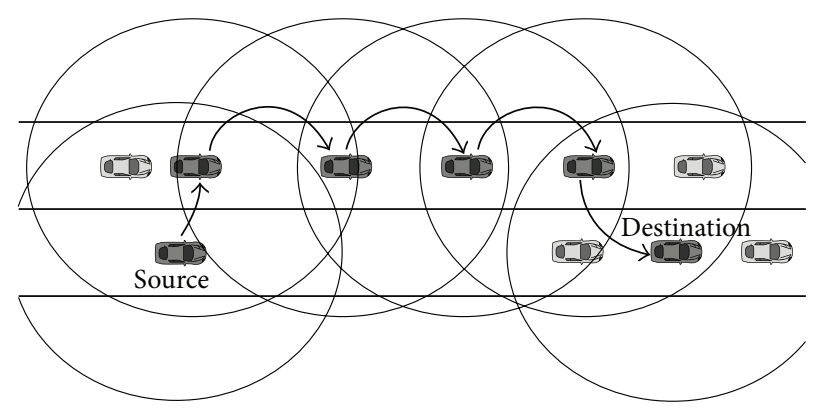

FIgURE 3: Perimeter forwarding in GPSR when employed in vehicular networks.

its range, even though this vehicle is still listed in the source vehicle's list of neighbours. Such wrong forwarding decisions lead to packet loss [43]. Hence, reliable one-hop neighbour nodes should be selected for greedy forwarding.

4.2. Design of Reliability Based GPSR-R Protocol. As in GPSR, we assume that all the vehicles that participate in the data transfer process possess the GPS facility to identify their location coordinates. The vehicles periodically transmit beacon messages to all the one-hop neighbours. In the proposed protocol, we modify the GPSR beacon frame by adding the following additional fields: (i) speed that contains the current velocity of the vehicle that generates the beacon; (ii) direction that contains the direction of movement of the vehicle that generates the beacon. The modified beacon structure is shown in Figure 4. On receiving the beacons from the neighbours, a tagged vehicle will be able to know the position of its neighbours as well as the velocity and direction with which these vehicles move. By using all these quantities and by using the results presented in Section 3 , the tagged vehicle computes the reliability of the communication link that is formed with each of its neighbour nodes. The vehicle then forms the neighbour list by including all one-hop neighbours, their ID's, and the corresponding link reliability probability values. The tagged vehicle also sets the beacon timer for all the vehicles in the neighbour list. Since the tagged vehicle receives the beacon message from its one-hop neighbours periodically, the neighbour list and the link reliability values also get updated periodically. At any point of time, if the tagged vehicle does not receive beacon message from a vehicle that is already included in the neighbour list, it assumes that this neighbour has gone out of its communication range and subsequently removes it from the list of neighbours. 


\begin{tabular}{|l|l|l|l|l|}
\hline Node address & $X$ coordinates & $Y$ coordinates & Velocity & Direction \\
\hline
\end{tabular}

FIGURE 4: Modified beacon structure in GPSR protocol.

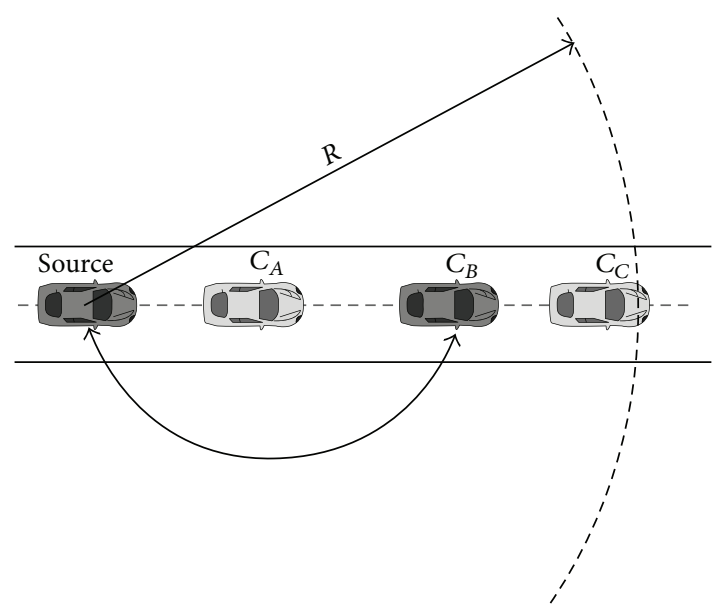

FIGURE 5: When reliability factor is considered in greedy forwarding.

TABLE 1: System parameters.

\begin{tabular}{lc}
\hline Packet size & 512 (variable) \\
MAC header & $272 \mathrm{bits}$ \\
PHY header & $128 \mathrm{bits}$ \\
ACK & $240 \mathrm{bits}$ \\
Channel bit rate & $2 \mathrm{Mbps}$ \\
Propagation delay & $2 \mu \mathrm{sec}$ \\
Slot time & $50 \mu \mathrm{sec}$ \\
SIFS & $28 \mu \mathrm{sec}$ \\
DIFS & $128 \mu \mathrm{sec}$ \\
Simulation time & $200 \mathrm{sec}$ \\
\hline
\end{tabular}

Whenever new vehicles enter the transmission range of the tagged vehicle, the neighbour list gets updated with the corresponding link reliability.

Figure 5 shows how forwarding will happen in the proposed reliability based GPSR protocol. Assume that the source vehicle has a data packet. In GPSR, the greedy forwarding algorithm will select vehicle $C_{C}$ as the forwarding node. However, there is a high probability that vehicle $C_{C}$ would leave the transmission range of the source vehicle even before it gets the data packet, which leads to packet loss. In the proposed scheme, a forwarding node is selected based on the reliability of the corresponding communication link. Given a set of vehicles that satisfies a requirement on link reliability, the vehicle that is closer to the destination acts as the forwarding vehicle. Figure 6 shows the flowchart for the proposed reliability based GPSR. Upon receiving a data packet for forwarding, the tagged vehicle checks whether the received data packet is in greedy or in perimeter mode. If the packet is in greedy mode, the tagged vehicle searches its neighbour table to identify the set of vehicles that satisfies the link reliability criterion. A vehicle belonging to this set that is geographically closer to the packet's destination is selected as the forwarding node. When the set of neighbours that satisfies the link reliability criterion is empty, the tagged vehicle marks the packet to perimeter mode. For the performance evaluation of the proposed protocol, we keep the reliability threshold to be equal to 0.6 . When the reliability threshold is too high, only limited number of vehicles will be available for forwarding, which increases the chances for the packet to enter perimeter forwarding mode. This will result in an increase of delay. Keeping very low values for the reliability threshold cannot significantly improve the protocol performance as compared to the conventional GPSR.

\section{Simulation Results}

In this section we present the results of our investigation. We evaluate the performance of proposed routing protocol and compare it with that of conventional GPSR protocol. We use the Network Simulator 2.33 (NS2.33) to conduct simulation experiments. Our simulation has two components: a mobility simulator and a wireless network simulator, which are connected by trace files that specify the vehicle mobility during simulation. A realistic vehicular mobility scenario is generated by using MOVE (mobility model generator for vehicular networks) [49] which is built on top of SUMO (Simulation of Urban Mobility) [50], which is an open source microtraffic simulation package. We construct a simulation area that uses a $10 \mathrm{~km}$ long highway with vehicles moving in the same direction. As described in Section 3, in the free flow traffic state, the vehicle speed and the traffic flow are independent and hence there are no significant interactions between the individual vehicles. Each vehicle is assigned a random velocity chosen from a uniform distribution. In general, we select the vehicle velocity to be uniform over $36 \mathrm{kmph}$, $108 \mathrm{kmph}$ with average value $72 \mathrm{kmph}$. The mobility trace file from MOVE contains information about realistic vehicle movements (such as their location, speed, and direction), which can be fed into discrete event simulators for network simulation. We record the trace files corresponding to vehicle mobility from SUMO, convert these files to NS2-compatible files using MOVE, and use them for network simulation using NS2.33. Each node in the network simulation represents one vehicle of the mobility simulations, moving according to the represented vehicles movement history in the trace file. IEEE 802.11 distributed coordination function is used as the MAC protocol. All the NS2 related settings are given in Table 1. For each simulation experiment, we perform ten runs to obtain the average results.

We assume that all the vehicles possess the GPS facility to identify their own location. As mentioned before, a tagged vehicle identifies the position of its neighbours through the exchange of one-hop beacon packets. In order to avoid synchronization of neighbour beacons, the beacons are transmitted in a time interval that is uniformly distributed over $0.5 B, 1.5 B$, where $B$ is the average interbeacon transmission time [7]. When a vehicle receives beacons from its neighbours, it sets the beacon timer for each of its neighbours so that the neighbour gets removed from the list when the corresponding beacon timer expires. In our experiment, we set the beacon timer to be equal to $4.5 B$ [7]. If $B$ is too 


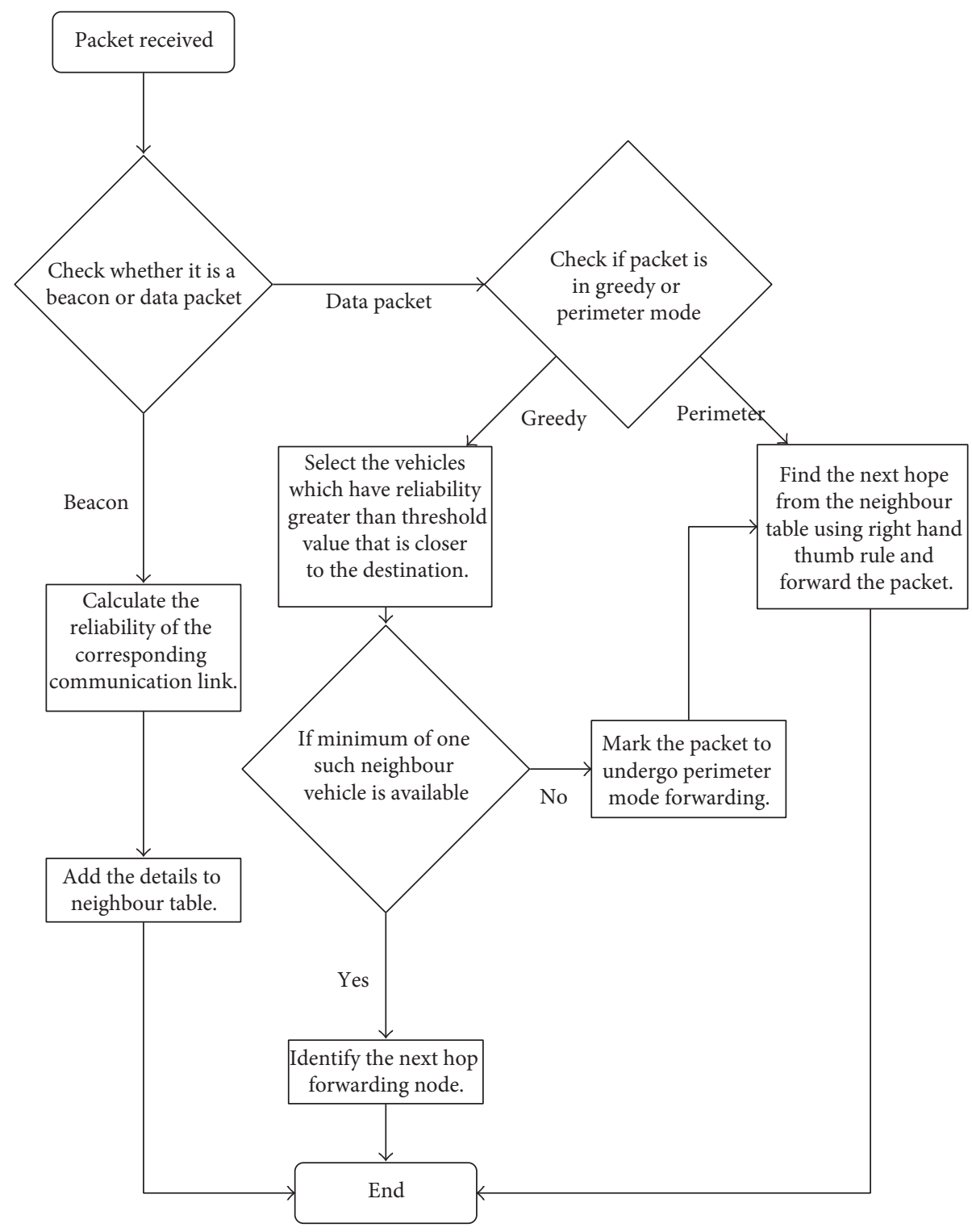

FIGURE 6: Flowchart of GPSR-R protocol.

small, then the neighbour table will be accurate but the congestion in the network will be high. If $B$ is too large, then the accuracy of the neighbour positions in the table will decrease. The correct value of $B$ depends on the mobility of the nodes and their communication range. We consider the data traffic to be CBR that is attached to each source vehicle to generate packets of fixed size. We further assume UDP as the transport layer protocol for the simulation studies. A total of 10 source-destination pairs are identified in the simulation which generates packets of size 512 bytes for every 0.25 seconds (we consider the case of variable packet size as well). Total time duration for the simulation is set as 200 seconds. The source vehicle will start generating the data packet after the first 10 seconds of the simulation time and stops generating the data packet at 150 seconds. For each simulation experiment, the sender/receiver node pairs are randomly selected. We consider the following performance metrics for the evaluation of the protocols.

Packet Delivery Ratio (PDR). This quantity is the average ratio of number of successfully received data packets at the destination vehicle to the number of packets generated by the source.

Average End-to-End (E2E) Delay. This is the time interval between receiving and sending time for a packet for a source to destination pair averaged over all such pairs. Here the data packets that are successfully delivered to destinations are only considered for the calculation.

Average Throughput. This quantity represents the average amount of data bits successfully delivered at the destination 


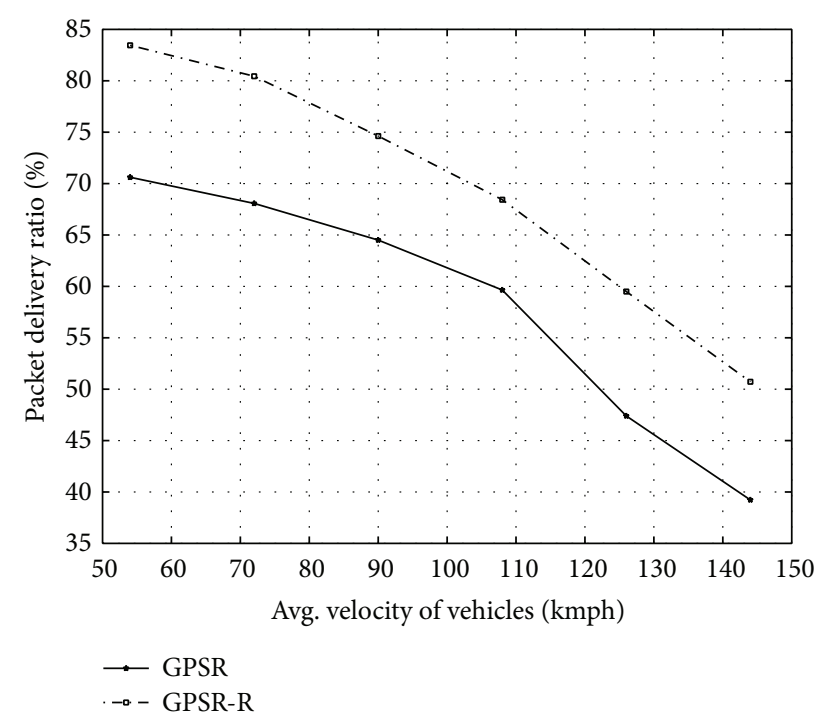

FIgURE 7: Average packet delivery ratio versus average velocity of vehicles.

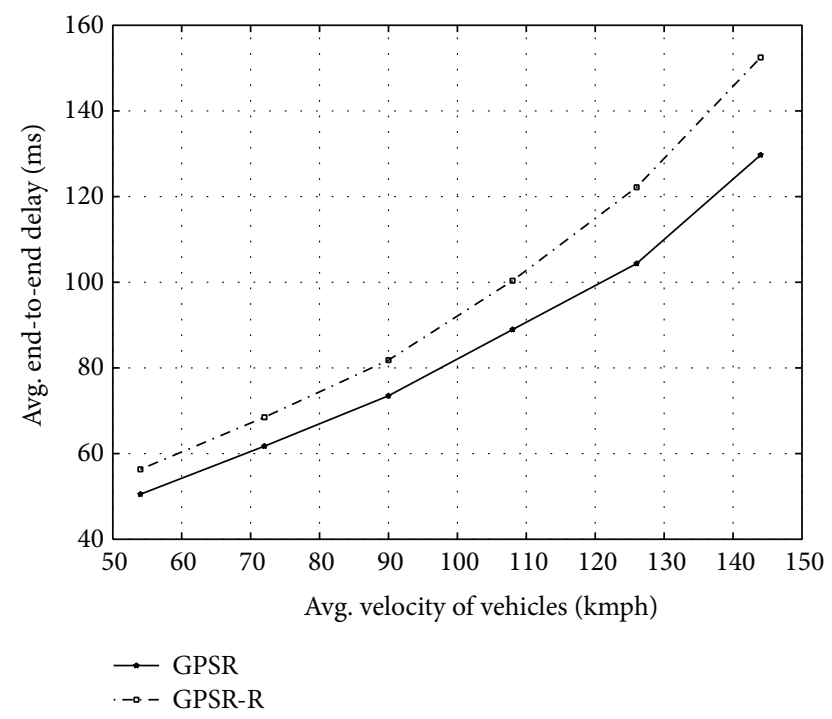

FIGURE 8: Average end-to-end delay versus different average vehicle velocity.

vehicle for a given source-destination pair average over all such pairs in the network.

We investigate the impact of average velocity on PDR for the proposed reliability based GPSR as well as for the conventional GPSR. In this case, we consider a total of 10 source-destination pairs which generate packets of size 512 bytes every 0.25 seconds. We set the vehicle's communication range to the default value equal to 250 meters. As shown in Figure 7, the average PDR reduces when the average velocity of the vehicles in the network increases. This reduction is due to the fact that the network topology gets changed frequently when the average velocity increases. In GPSR$\mathrm{R}$ protocol, a forwarding vehicle is chosen if and only if the reliability of the communication link with the source vehicle exceeds the minimum required. This reduces the probability of link breakages, resulting in improved packet delivery ratio. Figure 8 shows the impact of average velocity on average end-to-end delay for the GPSR as well as for the proposed GPSR-R protocol. As average velocity increases, the network becomes more dynamic in nature and chances of occurrence of link breakages increase. This increases the end-to-end delay for both protocols. Further, the proposed GPSR-R protocol shows higher average end-to-end delay than the GPSR protocol. The GPSR protocol selects the next hop vehicle by greedy forwarding in which a neighbour vehicle closest to the destination is selected as the next hop. However, in GPSR-R protocol, vehicles with reliability factor greater than the threshold form the set of next hop forwarding vehicles. Accordingly, the next hop forwarding vehicle selected need not be the one-hop vehicle closest to the destination. This results in higher number of hops to reach the destination and hence longer end-to-end delay. As shown in Figure 9, the average throughput of the network gets reduced when average velocity increases. As mentioned before, when the average velocity increases, the network topology gets changed frequently. This decreases the throughput.

We now investigate the impact of packet size on the performance of the two routing algorithms in VANETs. We vary the packet size from 512 bytes to 3072 bytes and keep the mean velocity of vehicles to be equal to $72 \mathrm{kmph}$. The PDR is plotted against packet size in Figure 10 while Figure 11 shows the variation of throughput. As packet size increases, there is a reduction in both the PDR and the throughput when GPSR protocol is employed. Notice that larger packets may be fragmented. If a fragmented data packet is lost during a link failure, then the whole data packet is lost. Accordingly, under GPSR, both the PDR and the throughput decrease when large size packets are employed. However, PDR and throughput performance of our proposed reliability based GPSR (GPSR$R$ ) are not significantly affected by varying packet size. This is because, in GPSR-R, one-hop forwarding vehicles are chosen based on reliability of the corresponding communication link. Hence the probability of link breakage is very less. Further, it can be observed that, in general, reliability based GPSR algorithm shows improvement in terms of PDR and throughput over the conventional GPSR. In Figure 12, the average end-to-end delay is plotted against the packet size. As explained earlier, the end-to-end delay for the reliable routing protocol GPSR-R is higher as compared to the conventional GPSR since, in GPSR-R, the next hop forwarding vehicle need not be the one closest to the destination. When the packet size exceeds limit, it gets fragmented into smaller size packets. If there is a link failure when a fragment is transmitted it affects the delivery of the fragmented packet. Accordingly, the delivery of the original packet also gets affected. Hence, in conventional GPSR, end-to-end delay increases as the packet size exceeds the fragmentation threshold. In the case of GPSR-R, since the forwarding nodes are selected based on link reliability criterion, the link breakage probability is less and hence there is high probability that all the fragments of a larger packet will be successfully delivered. Accordingly, the 


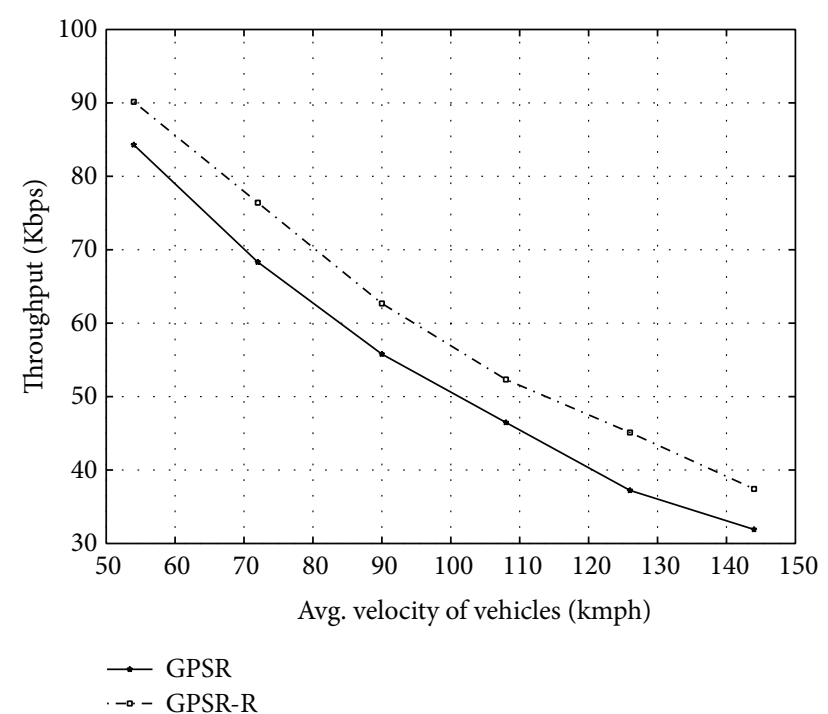

Figure 9: Average throughput versus different values of average velocity of the vehicles.

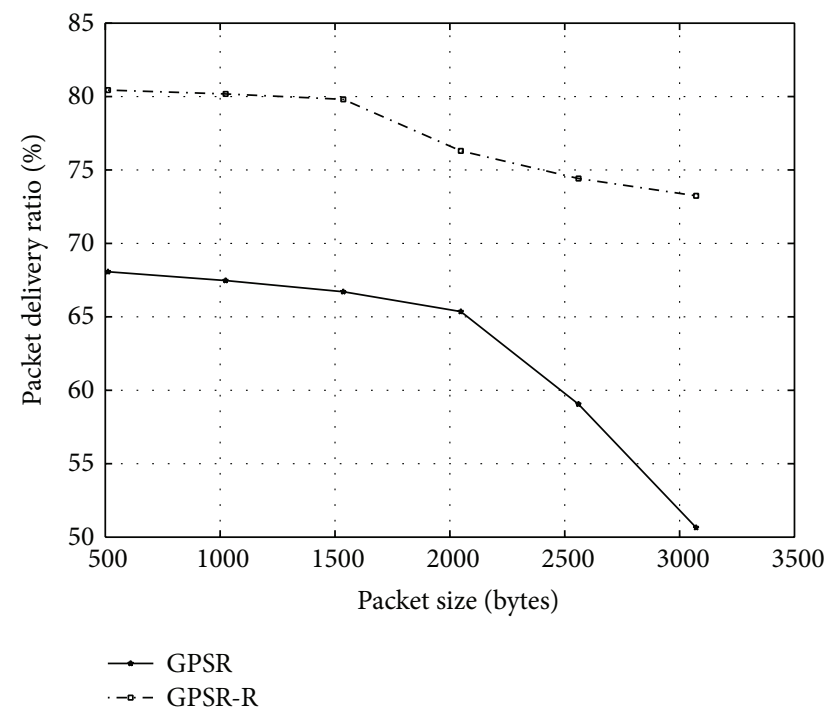

FIGURE 10: Average packet delivery ratio versus different packet size.

delay performance of GPSR-R is not affected significantly by varying packet size.

Next, we find the impact of varying the communication range of vehicles on the performance of the protocol. Figures 13 and 14, respectively, show the effect of range on PDR and throughput. As shown in Figures 13 and 14, both PDR and throughput increase when the communication range is increased. This happens because, with larger values of communication range and for a given value of vehicle density, there is a high probability for more numbers of vehicles in the neighbourhood of a tagged vehicle. Further, the PDR of reliability based GPSR is higher than that of the conventional GPSR owing to the fact that, in the former case, we consider link reliability as a metric for the selection of forwarding node. As shown in Figure 15, the average end-to-end delay

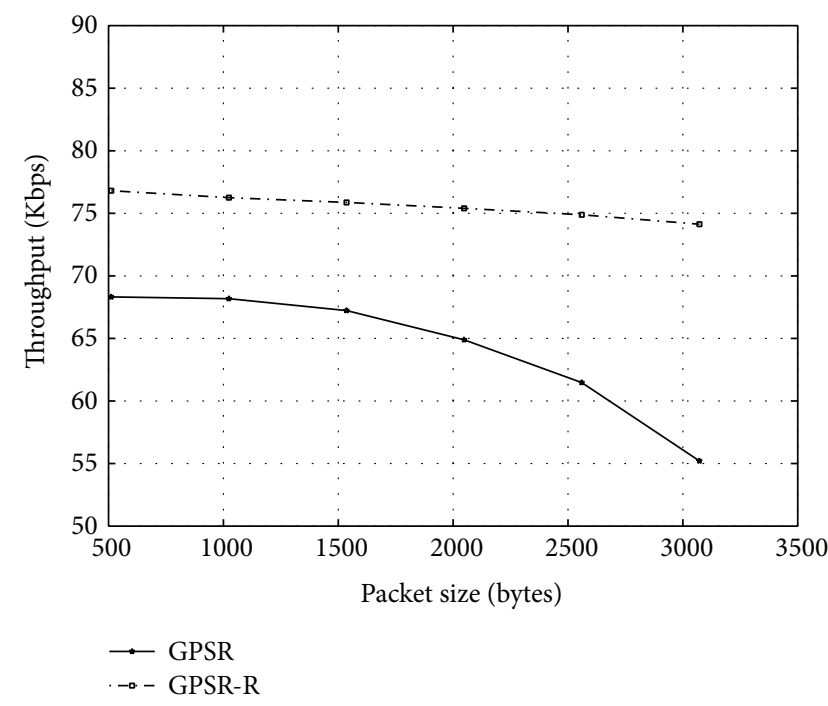

FIGURE 11: Average throughput versus different packet size.

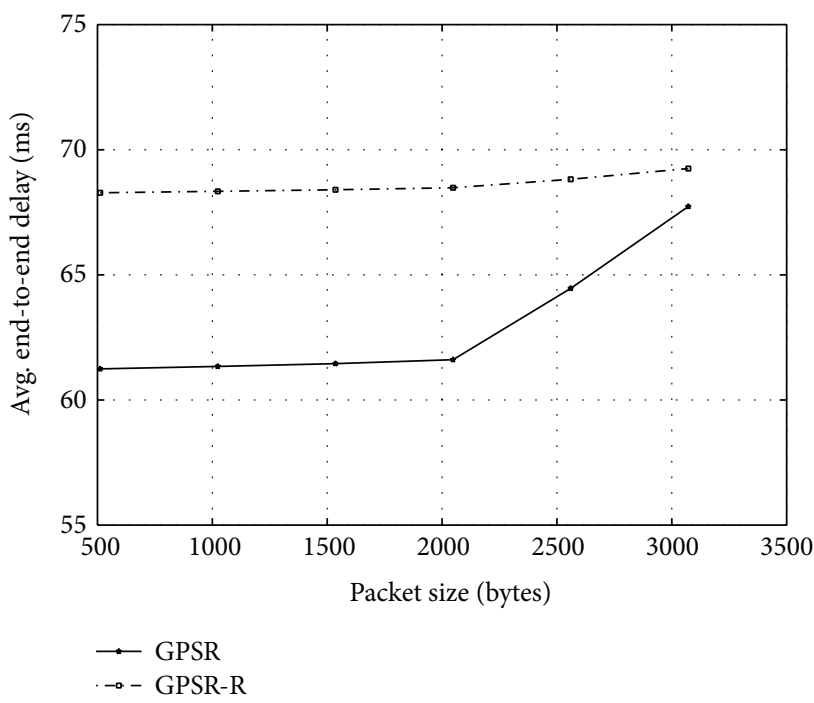

FIGURE 12: Average end-to-end delay versus different packet size.

decreases as the range is increased. In the case of conventional GPSR protocol, with larger values of communication range for tagged vehicle, there is a high probability for more numbers of vehicles to be available in the neighbourhood. Consequently, greedy forwarding is always possible and thus vehicles do not have to use perimeter forwarding which improves the delay performance. This is true in the case of GPSR-R as well. However, the selected forwarding node need not be the one close to the destination. This may result in an increase in the number of hops and hence longer end-to-end delay for GPSR-R protocol.

In Figure 16, we plot the PDR by varying the beacon interval time $(B)$. Here, we keep the average velocity as $72 \mathrm{kmph}$ and select the packet size as 512 bytes. As we increase $B$, the accuracy of the neighbour table decreases; that is, the positions of the neighbour nodes become more obsolete; this 


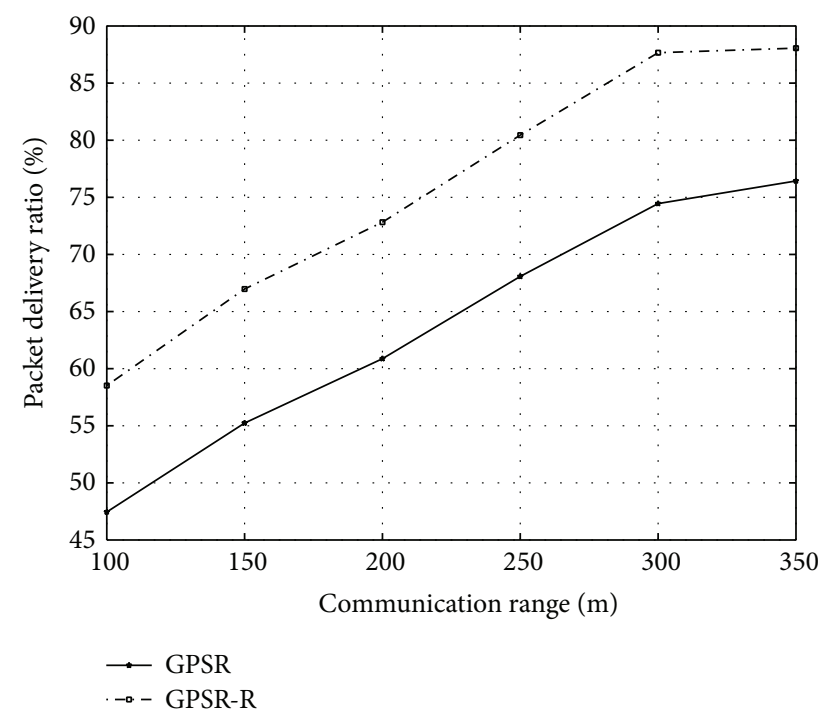

FIGURE 13: Average packet delivery ratio versus different communication range $R$.

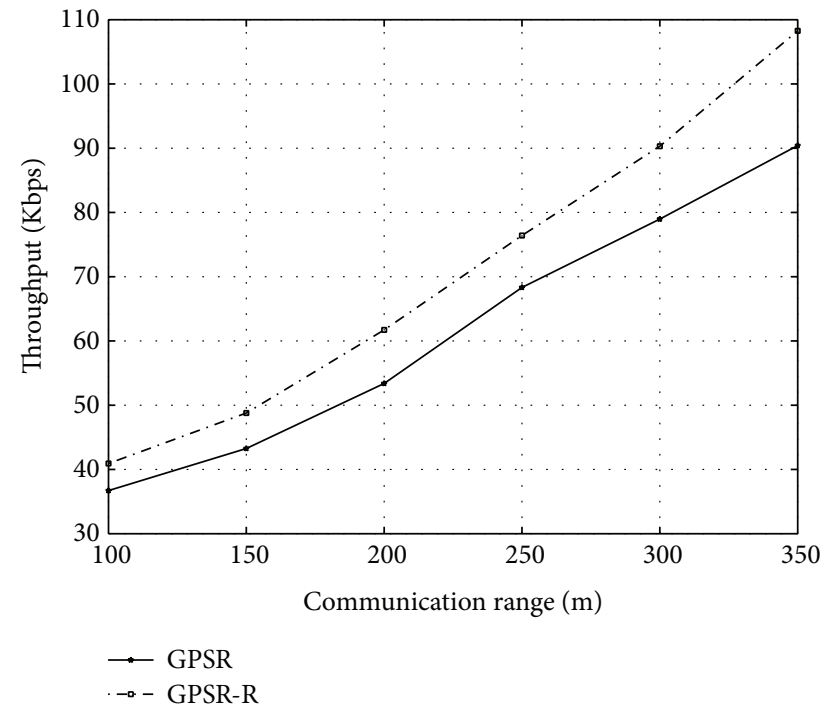

FIGURE 14: Average throughput versus different values of communication range.

increases the chances of link failures. Accordingly, the PDR decreases when the beacon interval is increased. However, for the proposed reliability based GPSR-R protocol, the rate of decrease of PDR has been observed to be less as compared to that of the conventional GPSR protocol because, in GPSR$\mathrm{R}$ protocol, vehicles with reliability factor greater than the threshold form the set of next hop forwarding vehicles.

In Figures 17-19, we compare the performance of our proposed protocol GPSR-R with that of conventional GPSR [7], GPSR-L [23], AODV-R [24], and MOPR-GPSR [20]. Figure 17 shows the comparison results for the packet delivery ratio of the network for all the abovementioned protocols. We select two distinct values for the average vehicle speed: $72 \mathrm{kmph}$ and $90 \mathrm{kmph}$. The simulation results show that our

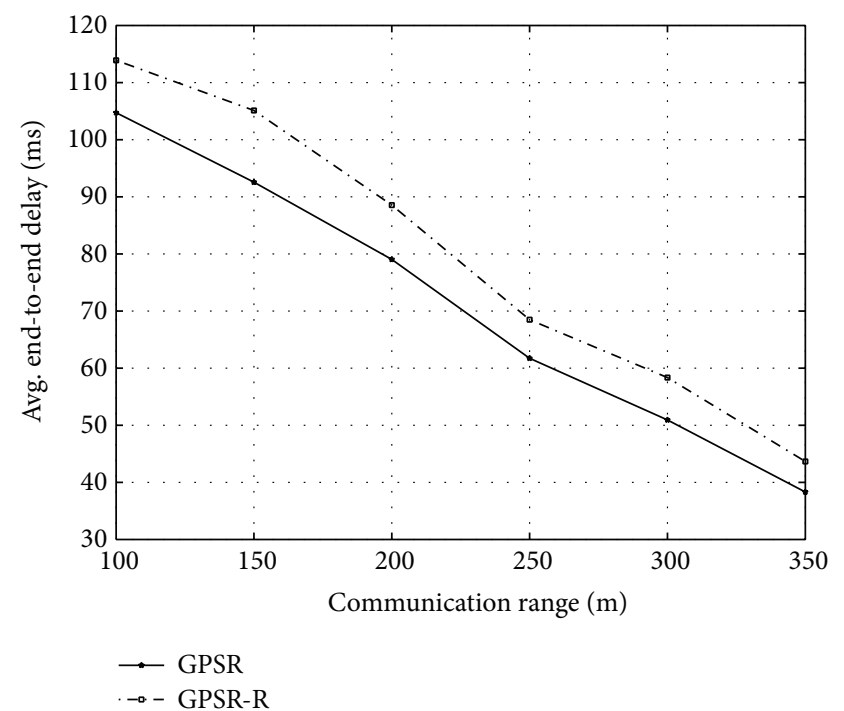

FIGURE 15: Average end-to-end delay versus different communication range $R$.

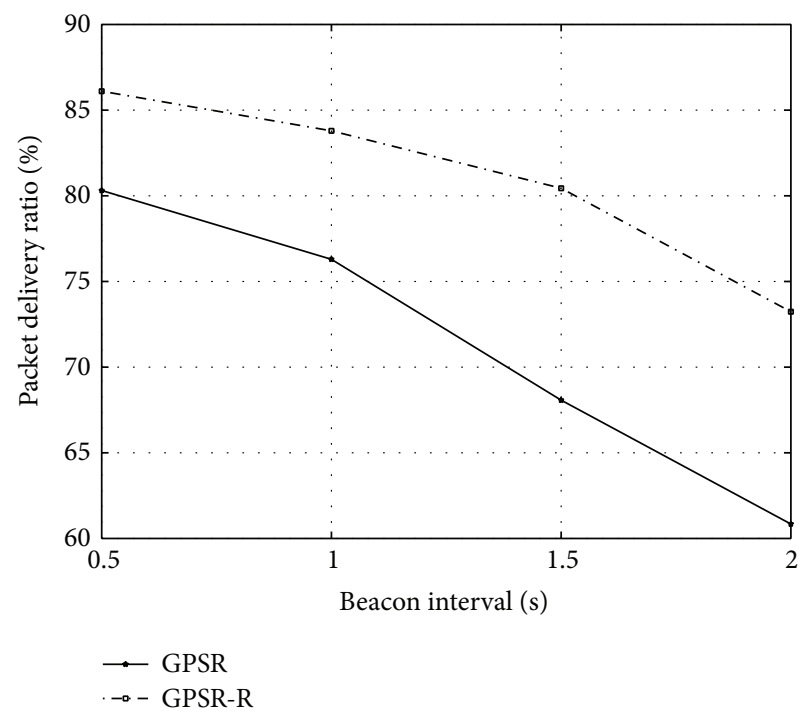

Figure 16: Packet delivery ratio versus different beacon interval period.

proposed routing scheme GPSR-R has the highest packet delivery ratio. At the same time, AODV-R gives the lowest packet delivery ratio compared to all other protocols under consideration, since topology based routing protocols such as AODV that require the exchange of several route requests and route reply messages are not suitable for high mobility applications. Figure 18 shows the comparison results for the network throughput when the abovementioned protocols are employed. Compared to all the protocols, the throughput of GPSR-R is higher. In the case of AODV-R the data transmission is jammed by the transmission of RREQ and RREP, which will decrease the average amount of data bits successfully delivered at the destination vehicle. Figure 19 shows the results for the average end-to-end delay experienced in 


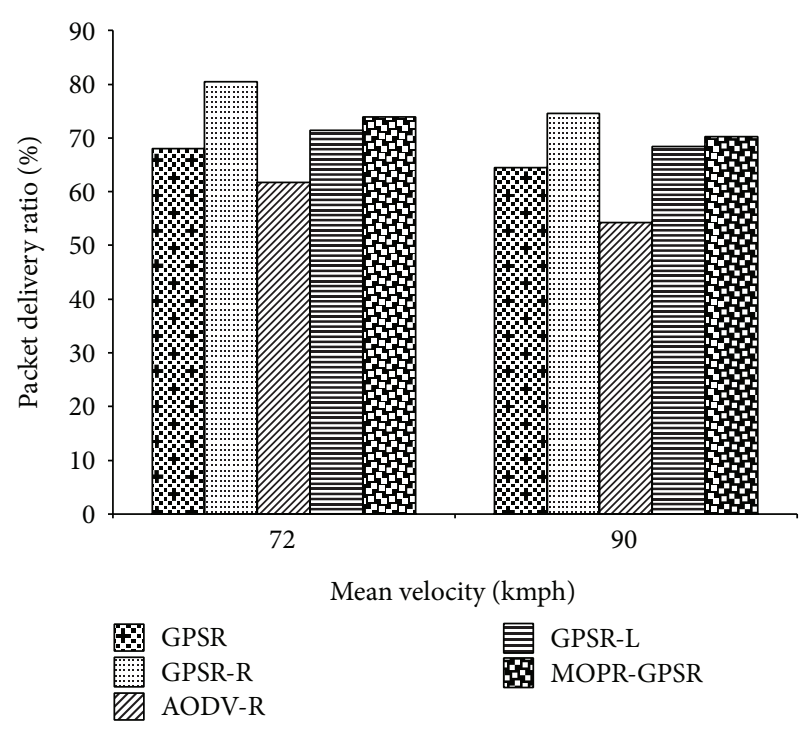

Figure 17: Packet delivery ratio comparison between various protocols.

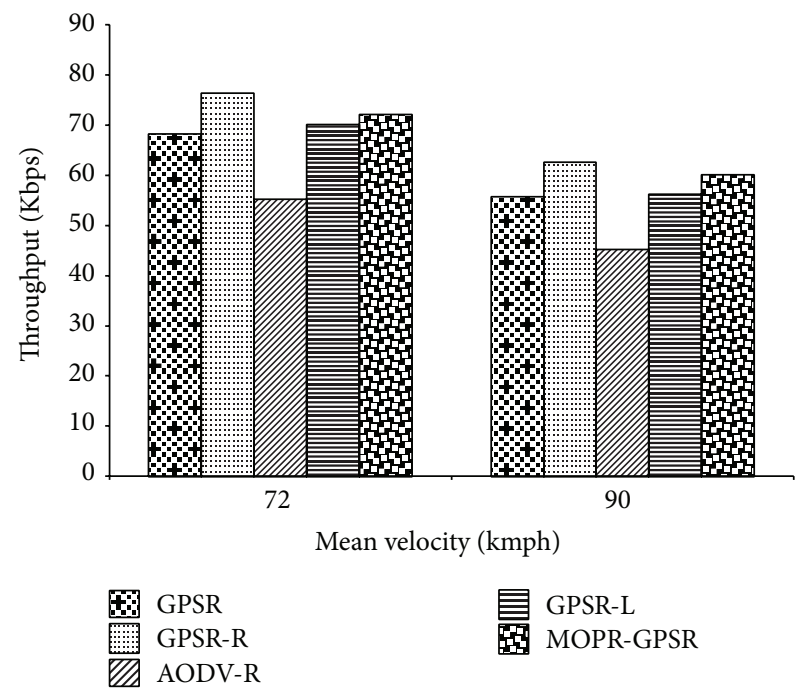

FIGURE 18: Throughput comparison between various protocols.

the network. AODV-R protocol suffers the highest delay compared to other protocols under consideration owing to the exchange of RREQ and RREP route request packets.

Further, the results show that the end-to-end delay is least for conventional GPSR protocol, since the packet is forwarded in a greedy forwarding manner in which a neighbour vehicle closest to the destination is selected as the next hop. Perimeter based forwarding will be followed if and only if greedy forwarding fails. The average end-to-end delay for GPSR-R, GPSR-L, and MOPR-GPSR will be slightly higher as compared to that of conventional GPSR since these GPSR enhancements do not follow greedy forwarding; instead they rely on stability of the links for the selection of forwarding vehicle. Accordingly, the next hop forwarding vehicle selected need not be the one-hop vehicle closest to

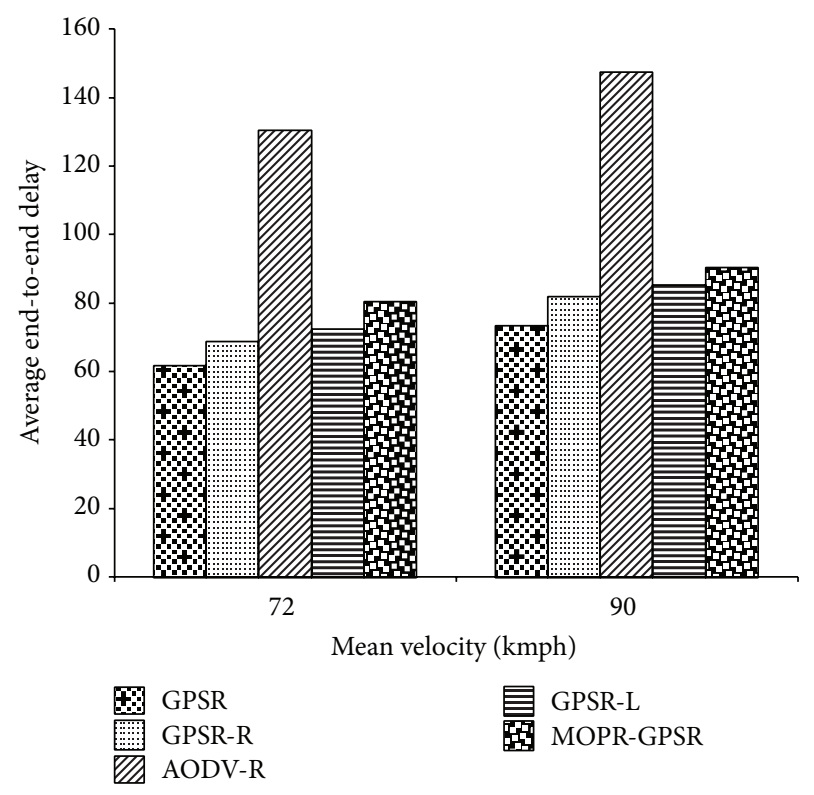

FIGURE 19: End-to-end delay comparison between various protocols.

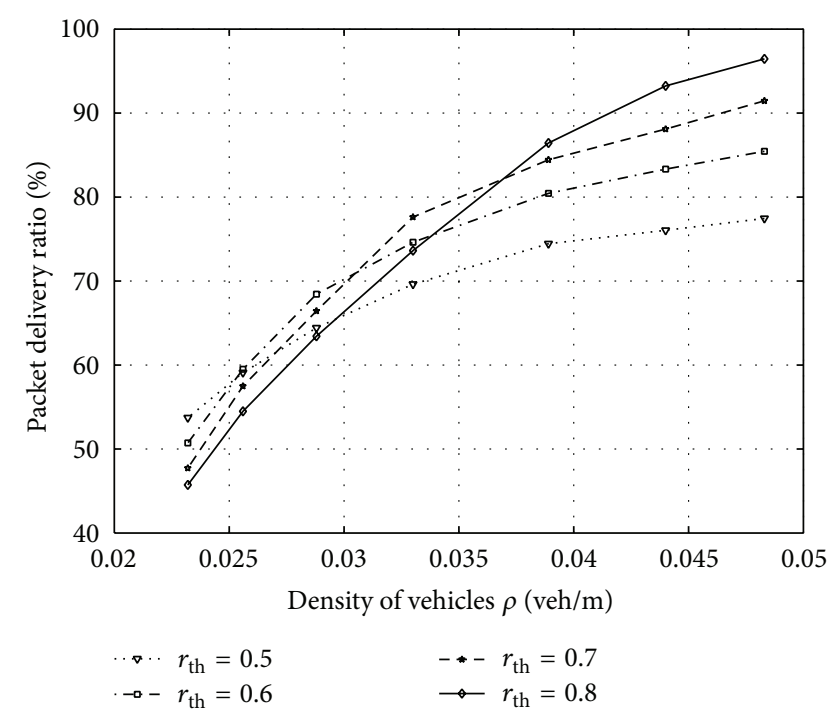

FIGURE 20: Average packet delivery ratio versus density of vehicles for the proposed link reliability based GPSR protocol.

the destination. This can result in higher number of hops to reach the destination and hence longer end-to-end delay. Hence, it can be concluded that even though the GPSR-L and MOPR-GPSR protocols show better results than AODV$\mathrm{R}$, the proposed routing protocol, GPSR-R, achieves the best performance in terms of network packet delivery ratio and throughput.

Figure 20 shows the PDR for the proposed GPSR-R protocol for various values of vehicle density. The PDR increases as the vehicle density is increased. The figure also shows the impact of selection of reliability threshold on PDR. When the vehicle density is comparatively smaller, keeping 


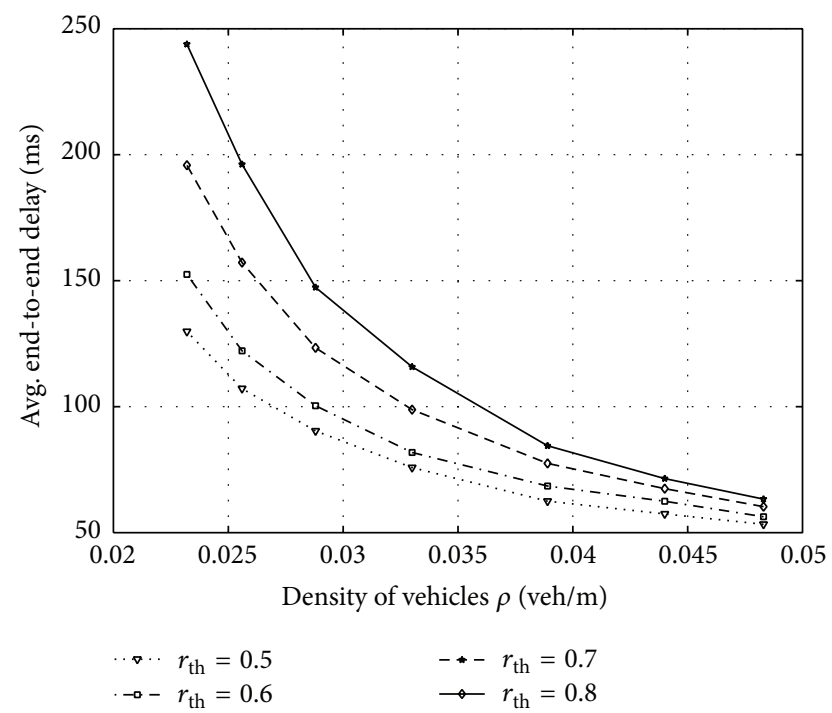

FIGURE 21: Average end-to-end delay versus density of vehicles for the proposed link reliability based GPSR protocol.

larger threshold reduces the PDR. This happens because of the nonavailability of potential forwarding nodes that meet the reliability criterion. When the vehicle density is increased, selection of larger threshold for the link reliability improves the PDR since links with higher reliability are chosen for forwarding the data. Figure 21 shows that end-to-end delay decreases as the vehicle density is increased since more vehicles will be available as forwarding nodes and probability of packet entering perimeter forwarding is less. In this case, keeping larger values for reliability threshold would increase the delay since the next hop vehicle selected as forwarding node need not be the one closest to the destination.

\section{Conclusion}

Designing reliable routing protocols for VANETs is quite a challenging task owing to the higher velocity of vehicles and mobility constraints on their movement in the network. In this paper, we have described a modification for the wellknown GPSR protocol, exploiting information about link reliability during selection of one-hop forwarding vehicles. In the proposed modified routing scheme, the vehicle that is closer to destination that satisfies the link reliability criterion will be selected as forwarding vehicle. We have also presented a probabilistic analysis of communication link reliability for one-dimensional VANETs and this model was used for the evaluation of the modified routing scheme. The proposed routing method ensures that most reliable nodes are chosen for forwarding and for building a route from source to destination. Through extensive simulation results, we have showed that the proposed protocol shows performance improvement over conventional GPSR protocol in terms of packet delivery ratio. Further, under the proposed scheme, the link failure rate is significantly reduced; however the delay slightly increases as compared to the conventional GPSR.

\section{Appendices}

\section{A. Derivation of (3)}

Since $V_{r}=V_{A}-V_{B}$, the dynamic range of $V_{r}$ is limited to $\left(-v_{m}, v_{m}\right)$. The $\operatorname{CDF} F_{V_{r}}\left(v_{r}\right)$ can be determined as follows:

$$
F_{V_{r}}\left(v_{r}\right)=P\left(V_{r} \leq v_{r}\right)=P\left(V_{A}-V_{B} \leq v_{r}\right) .
$$

Using the principle of random variable transformation [51], $F_{V_{r}}\left(v_{r}\right)$ can be determined as follows:

$$
F_{V_{r}}\left(v_{r}\right)=\left\{\begin{array}{l}
\int_{v_{A}=v_{\min }}^{v_{r}+v_{\max }} \int_{v_{B}=v_{A}-v_{r}}^{v_{\max }} f_{V_{A}, V_{B}}\left(v_{A}, v_{B}\right) \mathrm{d} v_{B} \mathrm{~d} v_{A} ; \\
-v_{m} \leq v_{r} \leq 0 \\
1-\int_{v_{A}=v_{r}+v_{\min }}^{v_{\max }} \int_{v_{B}=v_{\min }}^{v_{A}-v_{r}} f_{V_{A}, V_{B}}\left(v_{A}, v_{B}\right) \mathrm{d} v_{B} \mathrm{~d} v_{A} ; \\
0 \leq v_{r} \leq v_{m} .
\end{array}\right.
$$

The PDF of $V_{r}, f_{V_{r}}\left(v_{r}\right)$, is obtained by differentiating (A.2) with respect to $v_{r}$ and is given by

$$
f_{V_{r}}\left(v_{r}\right)=\left\{\begin{array}{l}
\frac{d}{d v_{r}} \int_{v_{A}=v_{\min }}^{v_{r}+v_{\max }} \int_{v_{B}=v_{A}-v_{r}}^{v_{\max }} f_{V_{A}, V_{B}}\left(v_{A}, v_{B}\right) \mathrm{d} v_{B} \mathrm{~d} v_{A} ; \\
-v_{m} \leq v_{r} \leq 0 \\
0-\frac{d}{d v_{r}} \\
\cdot \int_{v_{A}=v_{r}+v_{\min }}^{v_{\max }} \int_{v_{B}=v_{\min }}^{v_{A}-v_{r}} f_{V_{A}, v_{B}}\left(v_{A}, v_{B}\right) \mathrm{d} v_{B} \mathrm{~d} v_{A} ; \\
0 \leq v_{r} \leq v_{m} .
\end{array}\right.
$$

In the free flow traffic state, each vehicle moves independently of others. Further $V_{A}$ and $V_{B}$ are uniform over $v_{\min }$ and $v_{\max }$. Hence the above integral can be simplified to get the following expression for $f_{V_{r}}\left(v_{r}\right)$ :

$$
f_{V_{r}}\left(v_{r}\right)= \begin{cases}\frac{v_{r}+v_{\max }-v_{\min }}{\left(v_{\max }-v_{\min }\right)^{2}} ; & -v_{m} \leq v_{r} \leq 0 \\ \frac{v_{\max }-v_{\min }-v_{r}}{\left(v_{\max }-v_{\min }\right)^{2}} ; & 0 \leq v_{r} \leq v_{m} .\end{cases}
$$

\section{B. Derivation of $(\mathbf{8 a}),(8 b)$, and $(8 c)$}

From (6), the CDF of link duration $T, F_{T}(t)$, is given by

$$
F_{T}(t)=P(T \leq t)=P\left(X \leq V_{r} t\right) .
$$


Using the principle of random variable transformation [51], $F_{T}(t)$ can be determined as follows:

$$
F_{T^{(0)}}(t)=\left\{\begin{array}{c}
2 \int_{v_{r}=0}^{v_{m}} \int_{x=-R}^{v_{r} t} f_{X, V_{r}}\left(x, v_{r}\right) \mathrm{d} x \mathrm{~d} v_{r} ; \\
t \leq \frac{R}{v_{m}} \int_{v_{r}=0}^{v_{m}} \int_{x=-R}^{v_{r} t} f_{X, V_{r}}\left(x, v_{r}\right) \mathrm{d} x \mathrm{~d} v_{r} \\
-2 \int_{v_{r}=R / t}^{v_{m}} \int_{x=R}^{v_{r} t} f_{X, V_{r}}\left(x, v_{r}\right) \mathrm{d} x \mathrm{~d} v_{r} \\
t>\frac{R}{v_{m}},
\end{array}\right.
$$

where $R$ is the transmission range and $f_{X, V_{r}}\left(x, v_{r}\right)$ is the joint PDF of $X$ and $V_{r}$. The PDF of $T, f_{T}(t)$, is obtained by differentiating (B.2) with respect to $t$ and is given by

$$
f_{T}(t)= \begin{cases}f_{T_{1}}(t) ; & t \leq \frac{R}{v_{m}} \\ f_{T_{1}}(t)-f_{T_{2}}(t) ; & t>\frac{R}{v_{m}}\end{cases}
$$

where the components $f_{T_{1}}(t)$ and $f_{T_{2}}(t)$ correspond to the following two cases.

Case $1\left(t \leq R / v_{m}\right)$. Since $X$ and $V_{r}$ are independent random variables, $f_{T}(t)$ can be determined by substituting (3) and (5) into (B.2) and is given by the following expression represented as $f_{T_{1}}(t)$ :

$$
\begin{aligned}
f_{T_{1}}(t)= & 2 \frac{\rho}{\left(v_{\max }-v_{\min }\right)^{2}} \frac{d}{d t} \\
& \cdot \int_{v_{r}=0}^{v_{m}} \int_{x=-R}^{v_{r} t} e^{-\rho x}\left(\left(v_{\max }-v_{\min }\right)-v_{r}\right) \mathrm{d} x \mathrm{~d} v_{r} .
\end{aligned}
$$

The above integral can be simplified to get the following expression for $f_{T_{1}}(t)$ :

$$
f_{T_{1}}(t)=\rho \frac{\rho t v_{m}\left(e^{-\rho t v_{m}}+1\right)+2\left(e^{-\rho t v_{m}}-1\right)}{v_{m}^{2} \rho^{3} t^{3}} .
$$

Case 2 (when $t>R / v_{m}$ ). In this case, $f_{T}(t)$ is obtained by subtracting a component $f_{T_{2}}(t)$ from (B.5). Now $f_{T_{2}}(t)$ is determined by utilizing (3), (5), and (B.2):

$$
\begin{aligned}
f_{T_{2}}(t) & \\
= & 2 \frac{d}{d t} \int_{v_{r}=R / t}^{v_{m}} \int_{x=R}^{v_{r} t} f_{X}(x) f_{V_{r}}\left(v_{r}\right) \mathrm{d} x \mathrm{~d} v_{r} \\
= & 2 \frac{\rho}{\left(v_{\max }-v_{\min }\right)^{2}} \\
& \cdot \frac{d}{d t} \int_{v_{r}=R / t}^{v_{m}} \int_{x=R}^{v_{r} t} e^{-\rho x}\left(\left(v_{\max }-v_{\min }\right)-v_{r}\right) \mathrm{d} x \mathrm{~d} v_{r} .
\end{aligned}
$$

Notice that (B.6) can be simplified to get the following expression for $f_{T_{2}}(t)$ :

$$
\begin{gathered}
f_{T_{2}}(t)=\frac{1}{v_{m}^{2}}\left(\left(\frac{e^{-\rho t v_{m}}\left(\rho v_{m} t+2\right)}{\rho^{2} t^{3}}\right)-e^{-\rho R} R\left(\frac{v_{m}}{t^{2}}-\frac{R}{t^{3}}\right)\right. \\
\left.+e^{-\rho R}\left(\frac{v_{m}}{\rho t^{2}}-\frac{2 R}{\rho t^{3}}-\frac{2}{\rho^{2} t^{3}}\right)\right) .
\end{gathered}
$$

\section{Analytical Expression of (11)}

Analytical expression for link reliability probability given by (11) can be obtained by using (8a), (8b), and (8c). Substituting (8a), (8b), and (8c) into (11), we get final equation for the link reliability as follows:

$$
r_{\text {link }}(t)=\left\{\begin{array}{l}
\int_{t}^{t+T_{p}} f_{T_{1}}(t) d t \\
\quad\left(t+T_{P}\right) \leq \frac{R}{v_{m}} \\
\int_{t}^{t+T_{p}}\left(f_{T_{1}}(t)-f_{T_{2}}(t)\right) d t \\
t>\frac{R}{v_{m}} \\
\int_{t}^{R / v_{m}} f_{T_{1}}(t) d t+\int_{R / v_{m}}^{t+T_{p}}\left(f_{T_{1}}(t)-f_{T_{2}}(t)\right) d t \\
\quad\left(t+T_{p}\right)>\frac{R}{v_{m}}, t \leq \frac{R}{v_{m}} \\
0 ; \quad \\
T_{p} \leq 0 .
\end{array}\right.
$$


The various integral equations in (C.1) can be simplified using (8a), (8b), and (8c). The corresponding expressions (without substituting the limits) are given by

$$
\begin{aligned}
& \int f_{T_{1}}(t) d t=\left[\frac{-\rho v_{m} t E_{i}\left(-\rho v_{m} t\right)-e^{-\rho v_{m} t}}{\rho v_{m} t}\right. \\
& +\left(e ^ { - \rho v _ { m } t } \left(\rho^{2} v_{m}^{2} t^{2} e^{\rho v_{m} t} E_{i}\left(-\rho v_{m} t\right)\right.\right. \\
& \left.\left.+\rho v_{m} t-1\right)+1\right) \\
& \left.\left(v_{m}^{2} \rho^{2} t^{2}\right)^{-1}\right] \\
& \int f_{T_{2}}(t)=\frac{1}{v_{m}^{2}}\left[\frac{-v_{m}}{\rho} \frac{\rho v_{m} t E_{i}\left(-\rho v_{m} t\right)+e^{-\rho t v_{m}}}{t}\right. \\
& +e^{-\rho v_{m} t}\left(\rho^{2} v_{m}^{2} t^{2} e^{\rho v_{m} t} E_{i}\left(-\rho v_{m} t\right)\right. \\
& \left.+\rho v_{m} t-1\right) \cdot\left(\rho^{2} t^{2}\right)^{-1} \\
& -e^{\rho R} R\left(\frac{-v_{m}}{t}+\frac{R}{2 t^{2}}\right) \\
& \left.+e^{-\rho R}\left(\frac{-v_{m}}{\rho t}+\frac{R}{\rho t}+\frac{1}{\rho^{2} t^{2}}\right)\right] \text {, }
\end{aligned}
$$

where $E_{i}(-x)$ is the exponential integral given by [52]

$$
E_{i}(-x)=-\int_{x}^{\infty} \frac{e^{-t}}{t} \mathrm{~d} t
$$

Notice that the link reliability at time $t$ for a given prediction interval $T_{P}$ depends only on certain deterministic parameters, which makes it easy for the vehicles to compute this parameter online.

\section{Conflict of Interests}

The authors declare that there is no conflict of interests regarding the publication of this paper.

\section{References}

[1] J. J. Blum, A. Eskandarian, and L. J. Huffman, "Challenges of intervehicle Ad Hoc networks," IEEE Transactions on Intelligent Transportation Systems, vol. 5, no. 4, pp. 347-351, 2004.

[2] S. Zeadally, R. Hunt, Y.-S. Chen, A. Irwin, and A. Hassan, "Vehicular ad hoc networks (vanets): status, results, and challenges," Telecommunication Systems, vol. 50, no. 4, pp. 217-241, 2012.

[3] G. Karagiannis, O. Altintas, E. Ekici et al., "Vehicular networking: a survey and tutorial on requirements, architectures, challenges, standards and solutions," IEEE Communications Surveys and Tutorials, vol. 13, no. 4, pp. 584-616, 2011.

[4] A. V. Babu and V. K. M. Ajeer, "Analytical model for connectivity of vehicular ad hoc networks in the presence of channel randomness," International Journal of Communication Systems, vol. 26, no. 7, pp. 927-946, 2013.
[5] IEEE 802.11p/D5.0, "Draft amendment to standard for information technology telecommunications and information exchange between systems LAN/MAN specific requirements part 11: WLAN medium access control (MAC) and physical layer (PHY) specifications: wireless access in vehicular environments (WAVE)," 2008.

[6] F. Li and Y. Wang, "Routing in vehicular ad hoc networks: a survey," IEEE Vehicular Technology Magazine, vol. 2, no. 2, pp. 12-22, 2007.

[7] B. Karp and H. T. Kung, "GPSR: greedy perimeter stateless routing for wireless networks," in Proceedings of the 6th Annual International Conference on Mobile Computing and Networking (MOBICOM '00), pp. 243-254, August 2000.

[8] F. Granelli, G. Boato, D. Kliazovich, and G. Vernazza, "Enhanced GPSR routing in multi-hop vehicular communications through movement awareness," IEEE Communications Letters, vol. 11, no. 10, pp. 781-783, 2007.

[9] O. P. N. Perera and D. Jayalath, "Cross layer optimization of VANET routing with multi-objective decision making," in Proceedings of the Australasian Telecommunication Networks and Applications Conference (ATNAC '12), pp. 1-6, November 2012.

[10] Z. Ning, Y. Jung, Y. Jin, and K. C. Kim, "Route optimization for GPSR in VANET," in Proceedings of the IEEE International Advance Computing Conference (IACC'09), pp. 569-573, March 2009.

[11] H. Füßler, M. Mauve, H. Hartenstein, M. Käsemann, and D. Vollmer, "Mobicom poster: location-based routing for vehicular ad-hoc networks," ACM SIGMOBILE Mobile Computing and Communications Review, vol. 7, no. 1, pp. 47-49, 2003.

[12] S. Jiang, D. He, and J. Rao, "A prediction-based link availability estimation for mobile ad hoc networks," in Proceedings of the 20th Annual Joint Conference of the IEEE Computer and Communications Societies (INFOCOM '01), vol. 3, pp. 1745-1752, 2001.

[13] V. Thilagavathe and K. Duraiswamy, "Prediction based reliability estimation in manets with weibull nodes," European Journal of Scientific Research, vol. 64, no. 2, pp. 325-329, 2011.

[14] H.-M. Tsai, N. Wisitpongphan, and O. K. Tonguz, "Link-quality aware ad hoc on-demand distance vector routing protocol," in Proceedings of the 1st International Symposium on Wireless Pervasive Computing, pp. 6-12, 2006.

[15] D. Wu, R. Wang, and Y. Zhen, "Link stability-aware reliable packet transmitting mechanism in mobile ad hoc network," International Journal of Communication Systems, vol. 25, no. 12, pp. 1568-1584, 2012.

[16] T. Taleb, M. Ochi, A. Jamalipour, N. Kato, and Y. Nemoto, "An efficient vehicle-heading based routing protocol for VANET networks," in Proceedings of the IEEE Wireless Communications and Networking Conference (WCNC '06), vol. 4, pp. 2199-2204, April 2006.

[17] S. Wan, J. Tang, and R. S. Wolff, "Reliable routing for roadside to vehicle communications in rural areas," in Proceedings of the IEEE International Conference on Communications (ICC '08), pp. 3017-3021, IEEE, Beijing, China, May 2008.

[18] V. Namboodiri and L. Gao, "Prediction-based routing for vehicular Ad Hoc networks," IEEE Transactions on Vehicular Technology, vol. 56, no. 4, pp. 2332-2345, 2007.

[19] Menouar, M. Lenardi, and F. Filali, "A movement prediction based routing protocol for vehicle-to-vehicle communications," in Proceedings of the International Vehicle-to-Vehicle Communications Workshop (V2VCOM '05), vol. 21, pp. 7-14, 2005. 
[20] H. Menouarand, M. Lenardi, and F. Filali, "Movement Prediction-based Routing (MOPR) concept for position-based routing in vehicular networks," in Proceedings of the IEEE 66th Vehicular Technology Conference (VTC '07), pp. 2101-2105, October 2007.

[21] N. Sofra and K. K. Leung, "Link classification and residual time estimation through adaptive modeling for vanets," in Proceedings of the IEEE 69th Vehicular Technology Conference (VTC Spring '09), pp. 1-5, Barcelona, Spain, April 2009.

[22] N. Sofra, A. Gkelias, and K. K. Leung, "Route construction for long lifetime in vanets," IEEE Transactions on Vehicular Technology, vol. 60, no. 7, pp. 3450-3461, 2011.

[23] S. A. Rao, M. Pai, M. Boussedjra, and J. Mouzna, "Gpsr-L: greedy perimeter stateless routing with lifetime for vanets," in Proceedings of the 8th International Conference on ITS Telecommunications, pp. 299-304, 2008.

[24] M. H. Eiza, Q. Ni, T. Owens, and G. Min, "Investigation of routing reliability of vehicular ad hoc networks," EURASIP Journal on Wireless Communications and Networking, vol. 2013, no. 1, article 179, 2013.

[25] Z. Niu, W. Yao, Q. Ni, and Y. Song, "DeReQ: a QoS routing algorithm for multimedia communications in vehicular ad hoc networks," in Proceedings of the International Wireless Communications and Mobile Computing Conference (IWCMC '07), pp. 393-398, August 2007.

[26] X. Yu, H. Guo, and W.-C. Wong, "A reliable routing protocol for vanet communications," in Proceedings of the 7th International Wireless Communications and Mobile Computing Conference (IWCMC '11), pp. 1748-1753, July 2011.

[27] M. H. Eiza and Q. Ni, "An evolving graph-based reliable routing scheme for VANETs," IEEE Transactions on Vehicular Technology, vol. 62, no. 4, pp. 1493-1504, 2013.

[28] V. Naumov and T. R. Gross, "Connectivity-aware routing (CAR) in vehicular ad-hoc networks," in Proceedings of the 26th IEEE International Conference on Computer Communications (INFOCOM '07), pp. 1919-1927, Anchorage, Alaska, USA, May 2007.

[29] G. A. Galaviz-Mosqueda, R. Aquino-Santos, S. Villarreal-Reyes, R. Rivera-Rodríguez, L. Villaseñor-González, and A. Edwards, "Reliable freestanding position-based routing in highway scenarios," Sensors, vol. 12, no. 11, pp. 14262-14291, 2012.

[30] A. Boukerche, C. Rezende, and R. W. Pazzi, "A link-reliabilitybased approach to providing QoS support for VANETs," in Proceedings of the IEEE International Conference on Communications (ICC '09), pp. 1-5, June 2009.

[31] H. Yu, S. Ahn, and J. Yoo, "A stable routing protocol for vehicles in urban environments," International Journal of Distributed Sensor Networks, vol. 2013, Article ID 759261, 9 pages, 2013.

[32] X. Cai, Y. He, C. Zhao, L. Zhu, and C. Li, "LSGO: link state aware geographic opportunistic routing protocol for VANETs," EURASIP Journal on Wireless Communications and Networking, vol. 2014, no. 1, article 96, 2014.

[33] H. Wu, R. Fujimoto, R. Guensler, and M. Hunter, "MDDV: a mobility-centric data dissemination algorithm for vehicular networks," in Proceedings of the 1st ACM International Workshop on Vehicular Ad Hoc Networks (VANET '04), pp. 47-56, 2004.

[34] H. Labiod, N. Ababneh, and M. García de la Fuente, "An efficient scalable trajectory based forwarding scheme for VANETs," in Proceedings of the 24th IEEE International Conference on Advanced Information Networking and Applications (AINA '10), pp. 600-606, April 2010.
[35] H. N. Dau and H. Labiod, "Opportunistic trajectory-based routing for v2v communications," in Proceedings of the IEEE 22nd International Symposium on Personal, Indoor and Mobile Radio Communications (PIMRC '11), pp. 783-787, September 2011.

[36] F. de Rango, F. Veltri, P. Fazio, and S. Marano, "Two-level trajectory-based routing protocol for vehicular ad hoc networks in freeway and Manhattan environments," Journal of Networks, vol. 4, no. 9, pp. 866-880, 2009.

[37] W. Sun, H. Yamaguchi, K. Yukimasa, and S. Kusumoto, "GVGrid: a QoS routing protocol for vehicular ad hoc networks," in Proceedings of the 14th IEEE International Workshop on Quality of Service (IWQoS '06), pp. 130-139, June 2006.

[38] G. Yan and S. Olariu, "A probabilistic analysis of link duration in vehicular ad hoc networks," IEEE Transactions on Intelligent Transportation Systems, vol. 12, no. 4, pp. 1227-1236, 2011.

[39] M. Rudack, M. Meincke, K. Jobmann, and M. Lott, "On traffic dynamical aspects of Inter Vehicle Communications (IVC)," in Proceedings of the IEEE 58th Vehicular Technology Conference (VTC '03), vol. 5, pp. 3368-3372, October 2003.

[40] S.-Y. Wang, "The effects of wireless transmission range on path lifetime in vehicle-formed mobile ad hoc networks on highways," in Proceedings of the IEEE International Conference on Communications (ICC '05), vol. 5, pp. 3177-3181, IEEE, May 2005.

[41] K. Abboud and W. Zhuang, "Stochastic analysis of a singlehop communication link in vehicular ad hoc networks," IEEE Intelligent Transportation Systems Magazine, vol. 15, no. 5, pp. 2297-2307, 2014.

[42] S. Shelly and A. V. Babu, "Analysis of link life time in vehicular ad hoc networks for free-flow traffic state," Wireless Personal Communications, vol. 75, no. 1, pp. 81-102, 2014.

[43] R. A. Alsaqour, M. S. Abdelhaq, and O. A. Alsukour, "Effect of network parameters on neighbor wireless link breaks in GPSR protocol and enhancement using mobility prediction model," EURASIP Journal on Wireless Communications and Networking, vol. 2012, article 171, 15 pages, 2012.

[44] S. Yousefi, E. Altman, R. El-Azouzi, and M. Fathy, "Analytical model for connectivity in vehicular Ad Hoc networks," IEEE Transactions on Vehicular Technology, vol. 57, no. 6, pp. 33413356, 2008.

[45] J. Wu, "Connectivity of mobile linear networks with dynamic node population and delay constraint," IEEE Journal on Selected Areas in Communications, vol. 27, no. 7, pp. 1218-1225, 2009.

[46] W. McShane and R. Roess, Traffic Engineering, Pearson Prentice Hall, 3rd edition, 2004.

[47] D. L. Gerlough and M. J. Huber, “Traffic flow theory," Tech. Rep., 1976.

[48] T. D. C. Little and A. Agarwal, "An information propagation scheme for vanets," in Proceedings of the 8th International IEEE Conference on Intelligent Transportation Systems, pp. 155-160, September 2005.

[49] F. K. Karnadi, Z. H. Mo, and K. C. Lan, "Rapid generation of realistic mobility models for VANET," in Proceedings of the IEEE Wireless Communications and Networking Conference (WCNC '07), pp. 2508-2513, March 2007.

[50] D. Krajzewicz, J. Erdmann, M. Behrisch, and L. Bieker, "Recent development and applications of sumo simulation of urban mobility," International Journal on Advances in Systems and Measurements, vol. 5, no. 3-4, pp. 128-138, 2012. 
[51] A. Papoulis and S. U. Pillai, Probability, Random Variables, and Stochastic Processes, Tata McGraw-Hill Education, Noida, India, 2002.

[52] A. Jeffrey and D. Zwillinger, Table of Integrals, Series, and Products, Elsevier, 2007. 

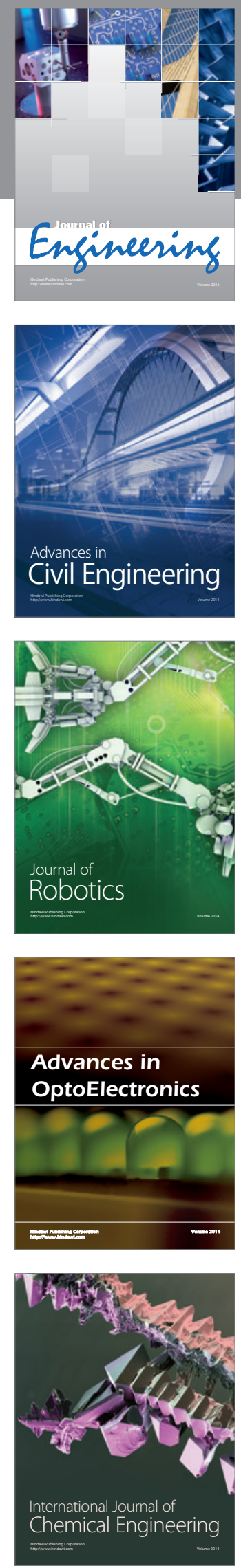

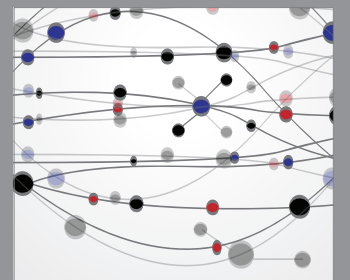

The Scientific World Journal
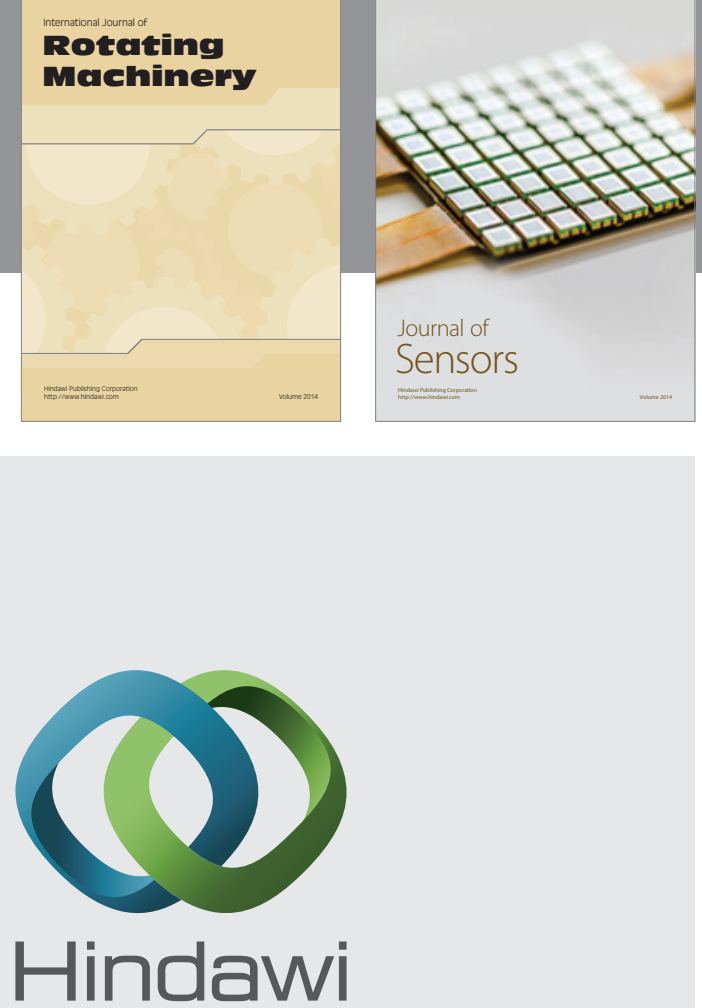

Submit your manuscripts at http://www.hindawi.com
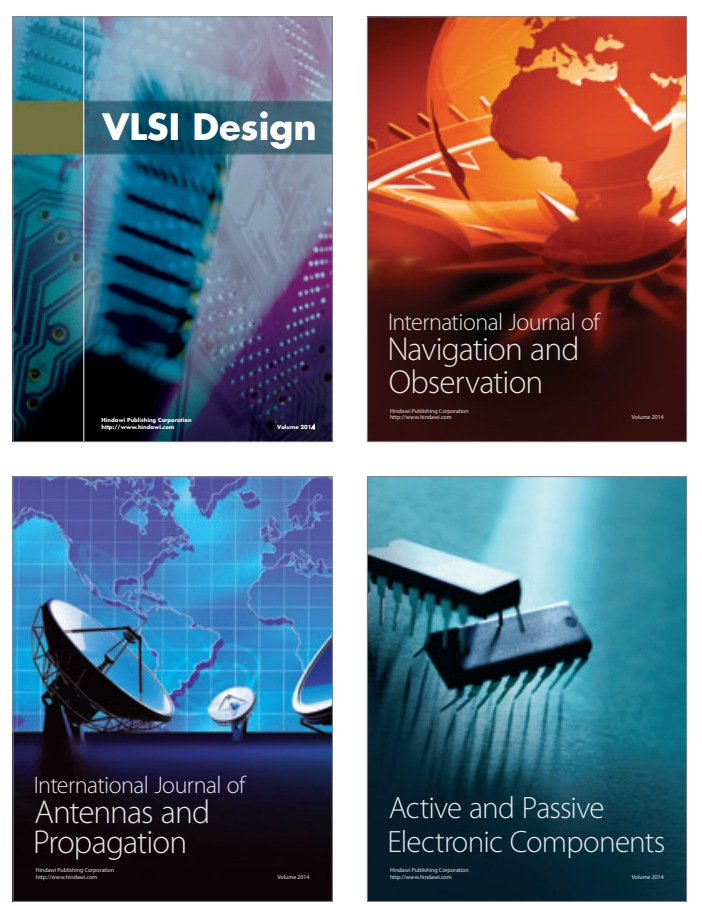
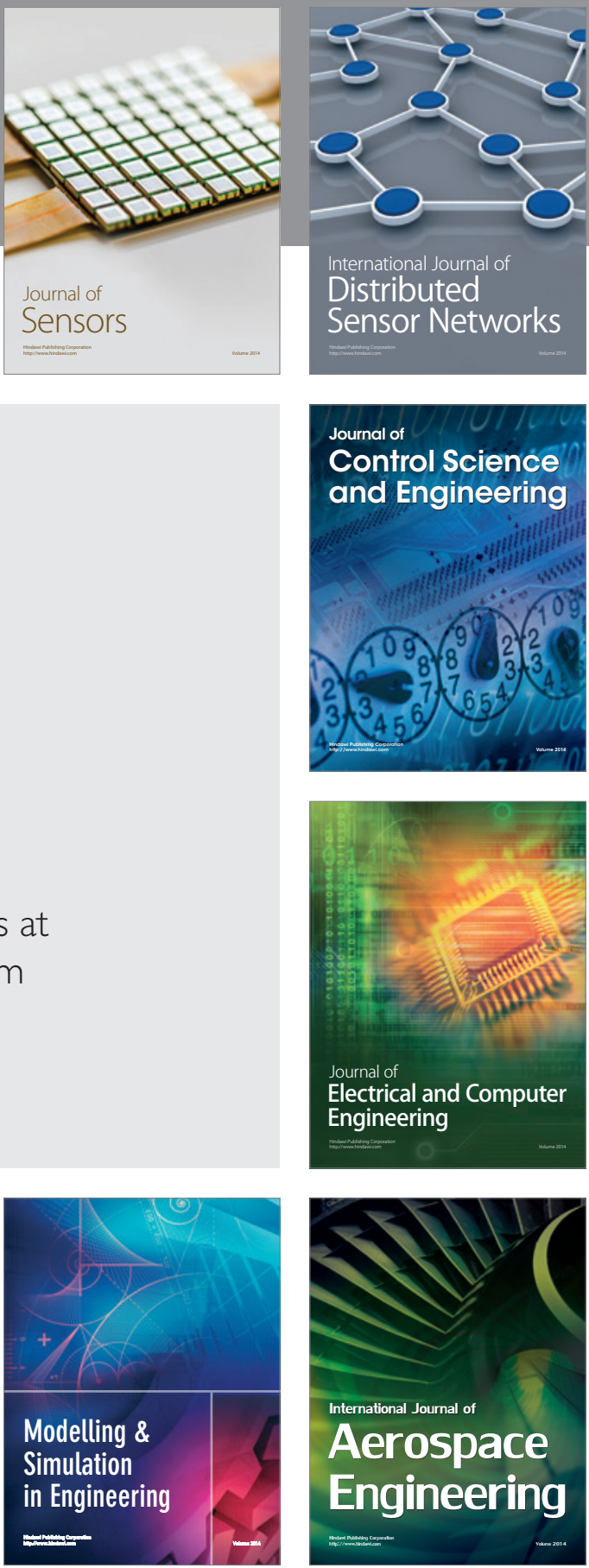

Journal of

Control Science

and Engineering
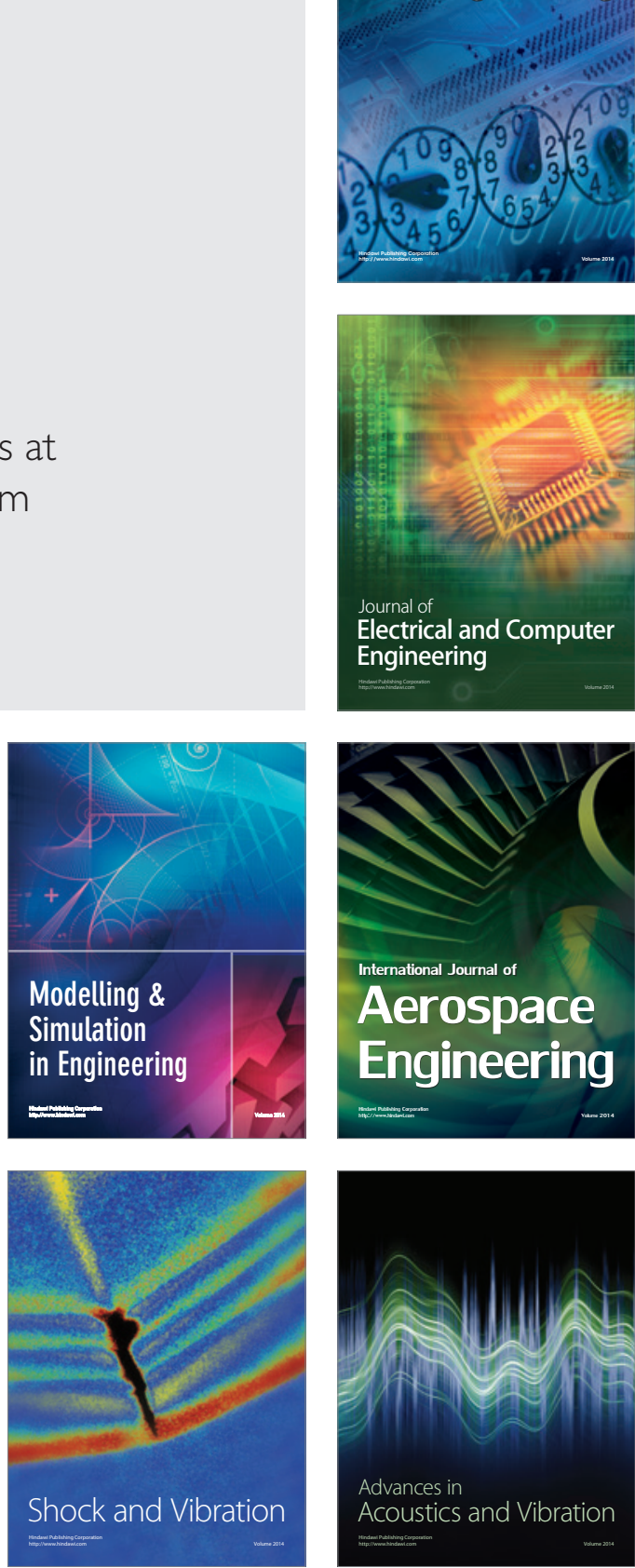